\title{
The Combinatorics of the Garsia-Haiman Modules for Hook Shapes
}

\author{
Ron M. Adin* \\ Department of Mathematics \\ Bar-Ilan University \\ Ramat-Gan 52900, Israel \\ radin@math.biu.ac.il
}

\author{
Jeffrey B. Remmel ${ }^{\dagger}$ \\ Department of Mathematics \\ University of California, San Diego \\ La Jolla, CA 92093 \\ remmel@math.ucsd.edu
}

\author{
Yuval Roichman $\ddagger$ \\ Department of Mathematics \\ Bar-Ilan University \\ Ramat-Gan 52900, Israel \\ yuvalr@math.biu.ac.il
}

Submitted: Feb 20, 2008; Accepted: Feb 28, 2008; Published: Mar 7, 2008

Mathematics Subject Classification: Primary 05E10, 13A50;

Secondary 05A19, 13F20, 20C30.

\begin{abstract}
Several bases of the Garsia-Haiman modules for hook shapes are given, as well as combinatorial decomposition rules for these modules. These bases and rules extend the classical ones for the coinvariant algebra of type $A$. We also give a decomposition of the Garsia-Haiman modules into descent representations.
\end{abstract}

\section{Introduction}

\subsection{Outline}

In [11], Garsia and Haiman introduced a module $\mathbf{H}_{\mu}$ for each partition $\mu$, which we shall call the Garsia-Haiman module for $\mu$. Garsia and Haiman introduced the modules $\mathbf{H}_{\mu}$ in

\footnotetext{
${ }^{*}$ Supported in part by the Israel Science Foundation, grant no. 947/04.

${ }^{\dagger}$ Supported in part by NSF grant DMS 0400507.

${ }^{\ddagger}$ Supported in part by the Israel Science Foundation, grant no. 947/04, and by the University of California, San Diego.
} 
attempt to prove Macdonald's $q$, $t$-Kostka polynomial conjecture and, in fact, the modules $\mathbf{H}_{\mu}$ played a major role in the resolution of Macdonald's conjecture [23]. When the shape $\mu$ has a single row, this module is isomorphic to the coinvariant algebra of type $A$. Our goal here is to understand the structure of this module when $\mu$ is a hook shape $\left(1^{k-1}, n-k+1\right)$.

A family of bases for the Garsia-Haiman module of hook shape $\left(1^{k-1}, n-k+1\right)$ is presented. This family includes the $k$-th Artin basis, the $k$-th descent basis, the $k$-th Haglund basis and the $k$-th Schubert basis as well as other bases. While the first basis appears in [13], the others are new and have interesting applications.

The $k$-th Haglund basis realizes Haglund's statistics for the modified Macdonald polynomials in the hook case. The $k$-th descent basis extends the well known Garsia-Stanton descent basis for the coinvariant algebra. The advantage of the $k$-th descent basis is that the $S_{n}$-action on it may be described explicitly. This description implies combinatorial rules for decomposing the bi-graded components of the module into Solomon descent representations and into irreducibles. In particular, a constructive proof of a formula due to Stembridge is deduced.

\section{Contents}

1 Introduction $\quad 1$

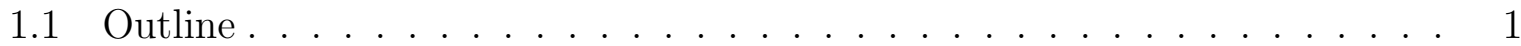

1.2 General Background . . . . . . . . . . . . . . . . . . . 3

1.3 Main Results - Bases . . . . . . . . . . . . . . . . . . . . 6

1.3.1 The $k$-th Descent Basis . . . . . . . . . . . . . . . . 6

1.3.2 The $k$-th Artin and Haglund Bases . . . . . . . . . . . . . . . 7

1.4 Main Results - Representations . . . . . . . . . . . . . . . . . 8

1.4.1 Decomposition into Descent Representations . . . . . . . . . . . 8

1.4.2 Decomposition into Irreducibles . . . . . . . . . . . . . . . 11

2 The Garsia-Haiman Module $\mathbf{H}_{\mu} \quad 13$

3 Generalized Kicking-Filtration Process 16

3.1 Proof of Theorem 1.5 . . . . . . . . . . . . . . . . 16

3.2 Applications . . . . . . . . . . . . . . . . . . . 19

4 A $k$-th Analogue of the Polynomial Ring 19

$4.1 \mathcal{P}_{n}^{(k)}$ and its Monomial Basis . . . . . . . . . . . . . . . . 20

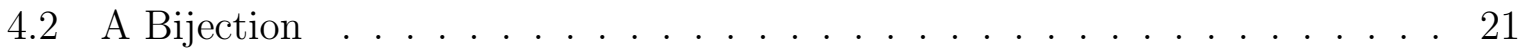

4.3 Action and Invariants . . . . . . . . . . . . . . . . . . 23

5 Straightening $\quad 24$

5.1 Basic Notions . . . . . . . . . . . . . . . . . . . . . . . 24

5.2 The Straightening Algorithm . . . . . . . . . . . . . . . . 26 
7 The Schur Function Expansion of $\tilde{H}_{\left(1^{k-1}, n-k+1\right)}(\bar{x} ; q, t) \quad 30$

7.1 Preliminaries . . . . . . . . . . . . . . . . 30

7.2 Second Proof of Theorem $1.16 \ldots \ldots \ldots \ldots$

8 Final Remarks $\quad 38$

8.1 Haglund Statistics _. . . . . . . . . . . . . . . . . . . 38

8.2 Relations with the Combinatorial Interpretation of Macdonald Polynomials 39

\subsection{General Background}

In 1988, I. G. Macdonald [27] introduced a remarkable new basis for the space of symmetric functions. The elements of this basis are denoted $P_{\lambda}(\bar{x} ; q, t)$, where $\lambda$ is a partition, $\bar{x}$ is a vector of indeterminates, and $q, t$ are parameters. The $P_{\lambda}(\bar{x} ; q, t)$ 's, which are now called "Macdonald polynomials", specialize to many of the well-known bases for the symmetric functions, by suitable substitutions for the parameters $q$ and $t$. In fact, we can obtain in this manner the Schur functions, the Hall-Littlewood symmetric functions, the Jack symmetric functions, the zonal symmetric functions, the zonal spherical functions, and the elementary and monomial symmetric functions.

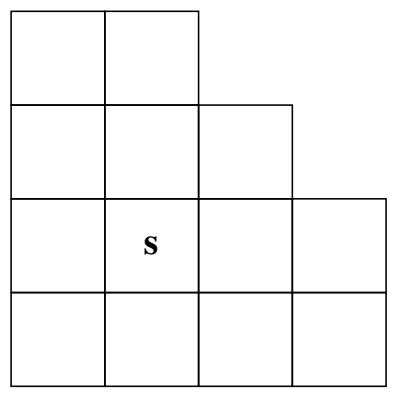

Figure 1: Diagram of a partition.

Given a cell $s$ in the Young diagram (drawn according to the French convention) of a partition $\lambda$, let $\operatorname{leg}_{\lambda}(s), \operatorname{leg}_{\lambda}^{\prime}(s), \operatorname{arm}_{\lambda}(s)$, and $\operatorname{arm}_{\lambda}^{\prime}(s)$ denote the number of squares that lie above, below, to the right, and to left of $s$ in $\lambda$, respectively. For example, when $\lambda=(2,3,3,4)$ and $s$ is the cell pictured in Figure 1, $\operatorname{leg}_{\lambda}(s)=2, \operatorname{leg}_{\lambda}^{\prime}(s)=1, \operatorname{arm}_{\lambda}(s)=2$ and $\operatorname{arm}_{\lambda}^{\prime}(s)=1$. For each partition $\lambda$, define

$$
h_{\lambda}(q, t):=\prod_{s \in \lambda}\left(1-q^{a r m_{\lambda}(s)} t^{\operatorname{leg}_{\lambda}(s)+1}\right)
$$

For a partition $\lambda=\left(\lambda_{1}, \ldots, \lambda_{k}\right)$ where $0<\lambda_{1} \leq \ldots \leq \lambda_{k}$, let $n(\lambda):=\sum_{i=1}^{k}(k-i) \lambda_{i}$. Macdonald introduced the $(q, t)$-Kostka polynomials $K_{\lambda, \mu}(q, t)$ via the equation

$$
J_{\mu}(\bar{x} ; q, t)=h_{\mu}(q, t) P_{\mu}(\bar{x} ; q, t)=\sum_{\lambda} K_{\lambda, \mu}(q, t) s_{\lambda}[X(1-t)],
$$


and conjectured that they are polynomials in $q$ and $t$ with non-negative integer coefficients.

In an attempt to prove Macdonald's conjecture, Garsia and Haiman [11] introduced the so-called modified Macdonald polynomials $\tilde{H}_{\mu}(\bar{x} ; q, t)$ as

$$
\tilde{H}_{\mu}(\bar{x} ; q, t)=\sum_{\lambda} \tilde{K}_{\lambda, \mu}(q, t) s_{\lambda}(\bar{x})
$$

where $\tilde{K}_{\lambda, \mu}(q, t):=t^{n(\mu)} K_{\lambda, \mu}(q, 1 / t)$. Their idea was that $\tilde{H}_{\mu}(\bar{x} ; q, t)$ is the Frobenius image of the character generating function of a certain bi-graded module $\mathbf{H}_{\mu}$ under the diagonal action of the symmetric group $S_{n}$. To define $\mathbf{H}_{\mu}$, assign (row, column)-coordinates to squares in the first quadrant, obtained by permuting the $(x, y)$ coordinates of the upper right-hand corner of the square so that the lower left-hand square has coordinates $(1,1)$, the square above it has coordinates $(2,1)$, the square to its right has coordinates $(1,2)$, etc. The first (row) coordinate of a square $w$ is denoted row $(w)$, and the second (column) coordinate of $w$ is the denoted $\operatorname{col}(w)$. Given a partition $\mu \vdash n$, let $\mu$ also denote the corresponding Young diagram, drawn according to the French convention, which consists of all the squares with coordinates $(i, j)$ such that $1 \leq i \leq \ell(\mu)$ and $1 \leq j \leq \mu_{i}$. For example, for $\mu=(2,2,4)$, the labelling of squares is depicted in Figure 2.

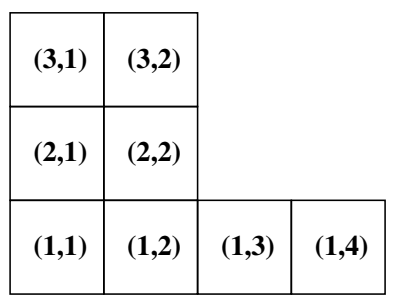

Figure 2: Labelling of the cells of a partition.

Fix an ordering $w_{1}, \ldots, w_{n}$ of the squares of $\mu$, and let

$$
\Delta_{\mu}\left(x_{1}, \ldots, x_{n} ; y_{1}, \ldots, y_{n}\right):=\operatorname{det}\left(x_{i}^{\operatorname{row}\left(w_{j}\right)-1} y_{i}^{\operatorname{col}\left(w_{j}\right)-1}\right)_{i, j} .
$$

For example,

$$
\Delta_{(2,2,4)}\left(x_{1}, \ldots, x_{8} ; y_{1}, \ldots, y_{8}\right)=\operatorname{det}\left(\begin{array}{cccccccc}
1 & y_{1} & y_{1}^{2} & y_{1}^{3} & x_{1} & x_{1} y_{1} & x_{1}^{2} & x_{1}^{2} y_{1} \\
1 & y_{2} & y_{2}^{2} & y_{2}^{3} & x_{2} & x_{2} y_{2} & x_{2}^{2} & x_{2}^{2} y_{2} \\
\vdots & & & & & & & \vdots \\
1 & y_{8} & y_{8}^{2} & y_{8}^{3} & x_{8} & x_{8} y_{8} & x_{8}^{2} & x_{8}^{2} y_{8}
\end{array}\right) \text {. }
$$

Now let $\mathbf{H}_{\mu}$ be the vector space of polynomials spanned by all the partial derivatives of $\Delta_{\mu}\left(x_{1}, \ldots, x_{n} ; y_{1}, \ldots, y_{n}\right)$. The symmetric group $S_{n}$ acts on $\mathbf{H}_{\mu}$ diagonally, where for any polynomial $P\left(x_{1}, \ldots, x_{n} ; y_{1}, \ldots, y_{n}\right)$ and any permutation $\sigma \in S_{n}$,

$$
P\left(x_{1}, \ldots, x_{n} ; y_{1}, \ldots, y_{n}\right)^{\sigma}:=P\left(x_{\sigma_{1}}, \ldots, x_{\sigma_{n}} ; y_{\sigma_{1}}, \ldots, y_{\sigma_{n}}\right) .
$$


The bi-degree $(h, k)$ of a monomial $x_{1}^{p_{1}} \cdots x_{n}^{p_{n}} y_{1}^{q_{1}} \cdots y_{n}^{q_{n}}$ is defined by $h:=\sum_{i=1}^{n} p_{i}$ and $k:=\sum_{i=1}^{n} q_{i}$. Let $\mathbf{H}_{\mu}^{(h, k)}$ denote space of homogeneous polynomials of degree $(h, k)$ in $\mathbf{H}_{\mu}$. Then

$$
\mathbf{H}_{\mu}=\bigoplus_{(h, k)} \mathbf{H}_{\mu}^{(h, k)} .
$$

The $S_{n}$-action clearly preserves the bi-degree so that $S_{n}$ acts on each homogeneous component $\mathbf{H}_{\mu}^{(h, k)}$. The character of the $S_{n}$-action on $\mathbf{H}_{\mu}^{(h, k)}$ can be decomposed as

$$
\chi^{\mathbf{H}_{\mu}^{(h, k)}}=\sum_{\lambda \vdash n} \chi_{\lambda, \mu}^{(h, k)} \chi^{\lambda},
$$

where $\chi^{\lambda}$ is the irreducible character of $S_{n}$ indexed by the partition $\lambda$ and the $\chi_{\lambda, \mu}^{(h, k)}$, s are non-negative integers. We then define the character generating function of $\mathbf{H}_{\mu}$ to be

$$
\begin{aligned}
\chi^{\mathbf{H}_{\mu}}(q, t) & =\sum_{h, k \geq 0} q^{h} t^{k} \sum_{\lambda \vdash|\mu|} \chi_{\lambda, \mu}^{(h, k)} \chi^{\lambda} \\
& =\sum_{\lambda \vdash|\mu|} \chi^{\lambda} \sum_{h, k \geq 0} \chi_{\lambda, \mu}^{(h, k)} q^{h} t^{k} .
\end{aligned}
$$

The Frobenius map $F$, which maps the center of the group algebra of $S_{n}$ to $\Lambda_{n}(\bar{x})$, is defined by sending the character $\chi^{\lambda}$ to the Schur function $s_{\lambda}(\bar{x})$. Garsia and Haiman conjectured that the Frobenius image of $\chi^{\mathbf{H}_{\mu}}(q, t)$ which they denoted by $F_{\mu}(q, t)$ is the modified Macdonald polynomial $\tilde{H}_{\mu}(\bar{x} ; q, t)$. That is, they conjectured that

$$
\begin{aligned}
F\left(\chi^{\mathbf{H}_{\mu}}(q, t)\right) & =\sum_{h, k \geq 0} q^{h} t^{k} \sum_{\lambda \vdash|\mu|} \chi_{\lambda, \mu}^{(h, k)} s_{\lambda}(\bar{x}) \\
& =\sum_{\lambda \vdash|\mu|} s_{\lambda}(\bar{x}) \sum_{h, k \geq 0} \chi_{\lambda, \mu}^{(h, k)} q^{h} t^{k} \\
& =\tilde{H}_{\mu}(\bar{x} ; q, t)
\end{aligned}
$$

so that

$$
\tilde{K}_{\lambda, \mu}(q, t)=\sum_{h, k \geq 0} \chi_{\lambda, \mu}^{(h, k)} q^{h} t^{k}
$$

Since Macdonald proved that $K_{\lambda, \mu}(1,1)=f_{\lambda}$, the number of standard tableau of shape $\lambda$, equations (7) and (8) led Garsia and Haiman [11] to conjecture that as an $S_{n}$-module, $\mathbf{H}_{\mu}$ carries the regular representation. This conjecture was eventually proved by Haiman [23] using the algebraic geometry of the Hilbert Scheme.

The goal of this paper is to understand the structure of the modules $\mathbf{H}_{\mu}$ when $\mu$ is a hook shape $\left(1^{k-1}, n-k+1\right)$. These modules were studied before by Stembridge [38], Garsia and Haiman [13], Allen [5] and Aval [6]. This paper suggests a detailed combinatorial analysis of the modules. 


\subsection{Main Results - Bases}

Consider the inner product $\langle$,$\rangle on the polynomial \operatorname{ring} \mathbf{Q}[\bar{x}, \bar{y}]=\mathbf{Q}\left[x_{1}, \ldots, x_{n}, y_{1}, \ldots, y_{n}\right]$ defined as follows:

$$
\langle f, g\rangle:=\text { constant term of } f\left(\partial_{x_{1}}, \ldots, \partial_{x_{n}} ; \partial_{y_{1}}, \ldots, \partial_{y_{n}}\right) g\left(x_{1}, \ldots, x_{n} ; y_{1}, \ldots, y_{n}\right) \text {, }
$$

where $f\left(\partial_{x_{1}}, \ldots, \partial y_{n}\right)$ is the differential operator obtained by replacing each variable $x_{i}$ $\left(y_{i}\right)$ in $f$ by the corresponding partial derivative $\frac{\partial}{\partial x_{i}}\left(\frac{\partial}{\partial y_{i}}\right)$. Let $J_{\mu}$ be the $S_{n}$-module dual to $\mathbf{H}_{\mu}$ with respect to $\langle$,$\rangle , and let \mathbf{H}_{\mu}^{\prime}:=\mathbf{Q}[\bar{x}, \bar{y}] / J_{\mu}$. It is not difficult to see that $\mathbf{H}_{\mu}$ and $\mathbf{H}_{\mu}^{\prime}$ are isomorphic as $S_{n}$-modules.

\subsubsection{The $k$-th Descent Basis}

The descent set of a permutation $\pi \in S_{n}$ is

$$
\operatorname{Des}(\pi):=\{i: \pi(i)>\pi(i+1)\}
$$

Garsia and Stanton [17] associated with each $\pi \in S_{n}$, the descent monomial

$$
a_{\pi}:=\prod_{i \in \operatorname{Des}(\pi)}\left(x_{\pi(1)} \cdots x_{\pi(i)}\right)=\prod_{j=1}^{n-1} x_{\pi(j)}^{|\operatorname{Des}(\pi) \cap\{j, \ldots, n-1\}|} .
$$

Using Stanley-Reisner rings, Garsia and Stanton [17] showed that the set $\left\{a_{\pi}: \pi \in S_{n}\right\}$ forms a basis for the coinvariant algebra of type $A$. See also [39] and [4].

Definition 1.1. For every integer $1 \leq k \leq n$ and permutation $\pi \in S_{n}$ define

$$
d_{i}^{(k)}(\pi):= \begin{cases}|\operatorname{Des}(\pi) \cap\{i, \ldots, k-1\}|, & \text { if } 1 \leq i<k \\ 0, & \text { if } i=k \\ |\operatorname{Des}(\pi) \cap\{k, \ldots, i-1\}|, & \text { if } k<i \leq n .\end{cases}
$$

Definition 1.2. For every integer $1 \leq k \leq n$ and permutation $\pi \in S_{n}$ define the $k$-th descent monomial

$$
\begin{aligned}
a_{\pi}^{(k)} & :=\prod_{\substack{i \in \operatorname{Des}(\pi) \\
i \leq k-1}}\left(x_{\pi(1)} \cdots x_{\pi(i)}\right) \cdot \prod_{\substack{i \in \operatorname{Des}(\pi) \\
i \geq k}}\left(y_{\pi(i+1)} \cdots y_{\pi(n)}\right) \\
& =\prod_{i=1}^{k-1} x_{\pi(i)}^{d_{i}^{(k)}(\pi)} \cdot \prod_{i=k+1}^{n} y_{\pi(i)}^{d_{i}^{(k)}(\pi)} .
\end{aligned}
$$

For example, if $n=8, k=4$, and $\pi=86147352$, then $\operatorname{Des}(\pi)=\{1,2,5,7\}$, $\left(d_{1}^{(4)}(\pi), \ldots, d_{8}^{(4)}(\pi)\right)=(2,1,0,0,0,1,1,2)$, and $a_{\pi}^{(4)}=x_{1}^{2} x_{2} y_{6} y_{7} y_{8}^{2}$.

Note that $a_{\pi}^{(n)}=a_{\pi}$, the Garsia-Stanton descent monomial.

Consider the partition $\mu=\left(1^{k-1}, n-k+1\right)$. 
Theorem 1.3. For every $1 \leq k \leq n$, the set of $k$-th descent monomials $\left\{a_{\pi}^{(k)}: \pi \in S_{n}\right\}$ forms a basis for the Garsia-Haiman module $\mathbf{H}_{\left(1^{k-1}, n-k+1\right)}$.

Two proofs of Theorem 1.3 are given in this paper. In Section 5 it is proved via a straightening algorithm. This proof implies an explicit description of the Garsia-Haiman hook module $\mathbf{H}_{\left(1^{k-1}, n-k+1\right)}^{\prime}$.

Theorem 1.4. For $\mu=\left(1^{k-1}, n-k+1\right)$ the ideal $J_{\mu}=\mathbf{H}_{\mu}^{\perp}$ defined above is the ideal of $\mathrm{Q}[\bar{x}, \bar{y}]$ generated by

(i) $\Lambda[\bar{x}]^{+}$and $\Lambda[\bar{y}]^{+}$(the symmetric functions in $\bar{x}$ and $\bar{y}$ without a constant term),

(ii) the monomials $x_{i_{1}} \cdots x_{i_{k}}\left(i_{1}<\cdots<i_{k}\right)$ and $y_{i_{1}} \cdots y_{i_{n-k+1}}\left(i_{1}<\cdots<i_{n-k+1}\right)$, and

(iii) the monomials $x_{i} y_{i}(1 \leq i \leq n)$.

This result has been obtained in a different form by J.-C. Aval [6, Theorem 2].

\subsubsection{The $k$-th Artin and Haglund Bases}

A second proof of Theorem 1.3 is given in Section 3. This proof applies a generalized version of the Garsia-Haiman kicking process. This construction is extended to a rich family of bases.

For every positive integer $n$, denote $[n]:=\{1, \ldots, n\}$. For every subset $A=\left\{i_{1}, \ldots, i_{k}\right\}$ $\subseteq[n]$ denote $\bar{x}_{A}:=x_{i_{1}}, \ldots, x_{i_{k}}$ and $\bar{y}_{A}:=y_{i_{1}}, \ldots, y_{i_{k}}$. Denote $\bar{x}:=\bar{x}_{[n]}=x_{1}, \ldots, x_{n}$ and $\bar{y}:=\bar{y}_{[n]}=y_{1}, \ldots, y_{n}$.

Let $k, c \in[n]$, let $A=\left\{a_{1}, \ldots, a_{k-1}\right\}$ be a subset of size $k-1$ of $[n] \backslash c$, and let $\bar{A}:=[n] \backslash(A \cup\{c\})$. Let $B_{A}$ be an arbitrary basis of the coinvariant algebra of $S_{k-1}$ acting on $\mathbf{Q}\left[\bar{x}_{A}\right]$, and let $C_{\bar{A}}$ be a basis of the coinvariant algebra of $S_{n-k}$ acting on $\mathbf{Q}\left[\bar{y}_{\bar{A}}\right]$. Finally define

$$
m_{(A, c, \bar{A})}:=\prod_{\{i \in A: i>c\}} x_{i} \prod_{\{j \in \bar{A}: j<c\}} y_{j} \in \mathbf{Q}[\bar{x}, \bar{y}] .
$$

Then

Theorem 1.5. The set

$$
\bigcup_{A, c} m_{(A, c, \bar{A})} B_{A} C_{\bar{A}}:=\bigcup_{A, c}\left\{m_{(A, c, \bar{A})} b c: b \in B_{A}, c \in C_{\bar{A}}\right\}
$$

forms a basis for the Garsia-Haiman module $\mathbf{H}_{\left(1^{k-1}, n-k+1\right)}^{\prime}$.

Definition 1.6. For every integer $1 \leq k \leq n$ and permutation $\pi \in S_{n}$ define

$$
\operatorname{inv}_{i}^{(k)}(\pi):= \begin{cases}\mid\{j: i<j \leq k \text { and } \pi(i)>\pi(j)\} \mid, & \text { if } 1 \leq i<k \\ 0, & \text { if } i=k \\ \mid\{j: k \leq j<i \text { and } \pi(j)>\pi(i)\} \mid, & \text { if } k<i \leq n .\end{cases}
$$


For every integer $1 \leq k \leq n$ and permutation $\pi \in S_{n}$ define the $k$-th Artin monomial

$$
b_{\pi}^{(k)}:=\prod_{i=1}^{k-1} x_{\pi(i)}^{\operatorname{inv}_{i}^{(k)}(\pi)} \cdot \prod_{i=k+1}^{n} y_{\pi(i)}^{\operatorname{inv}_{i}^{(k)}(\pi)} .
$$

and the $k$-th Haglund monomial

$$
c_{\pi}^{(k)}:=\prod_{i=1}^{k-1} x_{\pi(i)}^{d_{i}^{(k)}(\pi)} \cdot \prod_{i=k+1}^{n} y_{\pi(i)}^{\operatorname{inv}_{i}^{(k)}(\pi)} .
$$

For example, if $n=8, k=4$, and $\pi=86147352$, then $\operatorname{Des}(\pi)=\{1,2,5,7\}$, $\left(i n v_{1}^{(4)}(\pi), \ldots, i n v_{8}^{(4)}(\pi)\right)=(3,2,0,0,0,2,1,4), b_{\pi}^{(4)}=x_{8}^{3} x_{6}^{2} y_{3}^{2} y_{5} y_{2}^{4}$, and $c_{\pi}^{(4)}=x_{8}^{2} x_{6} y_{3}^{2} y_{5} y_{2}^{4}$.

Interesting special cases of Theorem 1.5 are the following.

Corollary 1.7. Each of the following sets :

$$
\left\{a_{\pi}^{(k)}: \pi \in S_{n}\right\}, \quad\left\{b_{\pi}^{(k)}: \pi \in S_{n}\right\}, \quad\left\{c_{\pi}^{(k)}: \pi \in S_{n}\right\}
$$

forms a basis for the Garsia-Haiman module $\mathbf{H}_{\left(1^{k-1}, n-k+1\right)}^{\prime}$.

\section{Remark 1.8.}

1. Garsia and Haiman [12] showed that $\left\{b_{\pi}^{(k)}: \pi \in S_{n}\right\}$ is a basis for $\mathbf{H}_{\left(1^{k-1}, n-k+1\right)}^{\prime}$. Other bases of $\mathbf{H}_{\left(1^{k-1}, n-k+1\right)}$ were also constructed by J-C Aval [6] and E. Allen [4, 5]. They used completely different methods. Aval constructed a basis of the form of an explicitly described set of partial differential operators applied to $\Delta_{\left(1^{k-1}, n-k+1\right)}$ and Allen constructed a basis for $\mathbf{H}_{\left(1^{k-1}, n-k+1\right)}$ out of his theory of bitableaux.

2. It should be noted that the last basis corresponds to Haglund's statistics for the Hilbert series of $\mathbf{H}_{\left(1^{k-1}, n-k+1\right)}$ that is implied by his combinatorial interpretation for the modified Macdonald polynomial $\tilde{H}_{\left(1^{k-1}, n-k+1\right)}(\bar{x} ; q, t)$; see Section 8 below.

3. Choosing $B_{A}$ and $C_{\bar{A}}$ in Theorem 1.5 to be the Schubert bases of the coinvariant algebras of $S_{k-1}$ (acting on $\mathbf{Q}\left[\bar{x}_{A}\right]$ ) and of $S_{n-k}$ (acting on $\mathbf{Q}\left[\bar{y}_{\bar{A}}\right]$ ), respectively, gives the $k$-th Schubert basis. One may study the Hecke algebra actions on this basis along the lines drawn in [2].

\subsection{Main Results - Representations}

\subsubsection{Decomposition into Descent Representations}

The set of elements in a Coxeter group having a fixed descent set carries a natural representation of the group, called a descent representation. Descent representations of Weyl groups were first introduced by Solomon [35] as alternating sums of permutation representations. This concept was extended to arbitrary Coxeter groups, using a different construction, by Kazhdan and Lusztig [25] [24, §7.15]. For Weyl groups of type A, 


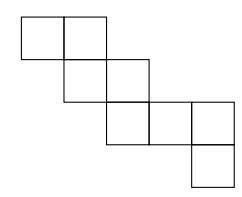

Figure 3: The zigzag shape corresponding to $n=8$ and $A=\{2,4,7\}$.

these representations also appear in the top homology of certain (Cohen-Macaulay) rankselected posets [37]. Another description (for type $A$ ) is by means of zig-zag diagrams $[18,16]$. A new construction of descent representations for Weyl groups of type $A$, using the coinvariant algebra as a representation space, is given in [1].

For every subset $A \subseteq\{1, \ldots, n-1\}$, let

$$
S_{n}^{A}:=\left\{\pi \in S_{n}: \operatorname{Des}(\pi)=A\right\}
$$

be the corresponding descent class and let $\rho^{A}$ denote the corresponding descent representation of $S_{n}$. Given $n$ and subset $A=\left\{a_{1}<\cdots<a_{k}\right\} \subseteq\{1, \ldots, n-1\}$, we can associate a composition of $n, \operatorname{comp}(A)=\left(c_{1}, \ldots, c_{k+1}\right)=\left(a_{1}, a_{2}-a_{1}, \ldots, a_{k}-a_{k-1}, n-a_{k}\right)$ and zigzig (skew) diagram $D_{A}$ which in French notation consists of rows of size $c_{1}, \ldots, c_{k+1}$, reading from top to bottom, which overlap by one square. For example, if $n=8$ and $A=\{2,4,7\}$, then $\operatorname{comp}(A)=(2,2,3,1)$ and $D(A)$ is the diagram pictured in Figure (3).

Definition 1.9. A bipartition (i.e., a pair of partitions) $\lambda=(\mu, \nu)$ where $\mu=\left(\mu_{1} \geq\right.$ $\left.\cdots \geq \mu_{k+1} \geq 0\right)$ and $\nu=\left(\nu_{1} \geq \cdots \geq \nu_{n-k+1} \geq 0\right)$ is called an $(n, k)$-bipartition if

1. $\lambda_{k}=\nu_{n-k+1}=0$ so that $\mu$ has at most $k-1$ parts and $\nu$ has at most $n-k$ parts, .

2. for $i=1, \ldots, k-1, \lambda_{i}-\lambda_{i+1} \in\{0,1\}$, and

3. for $i=1, \ldots, n-k, \nu_{i}-\nu_{i+1} \in\{0,1\}$.

For a permutation $\pi \in S_{n}$ and a corresponding $k$-descent basis element $a_{\pi}^{(k)}=\prod_{i=1}^{k-1} x_{\pi(i)}^{d_{i}}$. $\prod_{i=k+1}^{n} y_{\pi(i)}^{d_{i}}$, let

$$
\lambda\left(a_{\pi}^{(k)}\right):=\left(\left(d_{1}, d_{2}, \ldots, d_{k-1}, 0\right),\left(d_{n}, d_{n-1}, \ldots, d_{k+1}, 0\right)\right)
$$

be its exponent bipartition.

For an $(n, k)$-bipartition $\lambda=(\mu, \nu)$ let

$$
I_{\lambda}^{(k) \unlhd}:=\operatorname{span}_{\mathbf{Q}}\left\{a_{\pi}^{(k)}+J_{\left(1^{k-1}, n-k+1\right)}: \pi \in S_{n}, \lambda\left(a_{\pi}^{(k)}\right) \unlhd \lambda\right\},
$$

and

$$
I_{\lambda}^{(k) \triangleleft}:=\operatorname{span}_{\mathbf{Q}}\left\{a_{\pi}^{(k)}+J_{\left(1^{k-1}, n-k+1\right)}: \pi \in S_{n}, \lambda\left(a_{\pi}^{(k)}\right) \triangleleft \lambda\right\}
$$

be subspaces of the module $\mathbf{H}_{\left(1^{k-1}, n-k+1\right)}^{\prime}$, where $\unlhd$ is the dominance order on bipartitions (see Definition 5.4.1), and let

$$
R_{\lambda}^{(k)}:=I_{\lambda}^{(k) \unlhd} / I_{\lambda}^{(k) \triangleleft} .
$$


Proposition 1.10. $I_{\lambda}^{(k) \unlhd}, I_{\lambda}^{(k) \triangleleft}$ and thus $R_{\lambda}^{(k)}$ are $S_{n}$-invariant.

Lemma 1.11. Let $\lambda=(\mu, \nu)$ be an $(n, k)$-bipartition. Then

$$
\left\{a_{\pi}^{(k)}+I_{\lambda}^{(k) \triangleleft}: \operatorname{Des}(\pi)=A_{\lambda}\right\}
$$

is a basis for $R_{\lambda}^{(k)}$, where

$$
A_{\lambda}:=\left\{1 \leq i<n: \mu_{i}-\mu_{i+1}=1 \text { or } \nu_{n-i}-\nu_{n-i+1}=1\right\} .
$$

Theorem 1.12. The $S_{n}$-action on $R_{\lambda}^{(k)}$ is given by

$$
s_{j}\left(a_{\pi}^{(k)}\right)= \begin{cases}a_{s_{j} \pi}^{(k)}, & \text { if }\left|\pi^{-1}(j+1)-\pi^{-1}(j)\right|>1 ; \\ a_{\pi}^{(k)}, & \text { if } \pi^{-1}(j+1)=\pi^{-1}(j)+1 ; \\ -a_{\pi}^{(k)}-\sum_{\sigma \in A_{j}(\pi)} a_{\sigma}^{(k)}, & \text { if } \pi^{-1}(j+1)=\pi^{-1}(j)-1 .\end{cases}
$$

Here $s_{j}=(j, j+1)(1 \leq j<n)$ are the Coxeter generators of $S_{n}$ and $\left\{a_{\pi}^{(k)}+I_{\lambda}^{(k) \triangleleft}\right.$ : $\left.\operatorname{Des}(\pi)=A_{\lambda}\right\}$ is the descent basis of $R_{\lambda}^{(k)}$. For the definition of $A_{j}(\pi)$ see Theorem 6.4 below.

This explicit description of the action is then used to prove the following.

Theorem 1.13. Let $\lambda=(\mu, \nu)$ be an $(n, k)$-bipartition. $R_{\lambda}^{(k)}$ is isomorphic, as an $S_{n^{-}}$ module, to the Solomon descent representation determined by the descent class $\left\{\pi \in S_{n}\right.$ : $\left.\operatorname{Des}(\pi)=A_{\lambda}\right\}$.

Let $\mathbf{H}_{\left(1^{k-1}, n-k+1\right)}^{\prime\left(t_{1}, t_{2}\right)}$ be the $\left(t_{1}, t_{2}\right)$-th homogeneous component of $\mathbf{H}_{\left(1^{k-1}, n-k+1\right)}^{\prime}$.

Corollary 1.14. For every $t_{1}, t_{2} \geq 0$ and $1 \leq k \leq n$, the $\left(t_{1}, t_{2}\right)$-th homogeneous component of $\mathbf{H}_{\left(1^{k-1}, n-k+1\right)}^{\prime}$ decomposes into a direct sum of Solomon descent representations as follows:

$$
\mathbf{H}_{\left(1^{k-1}, n-k+1\right)}^{\left(t_{1}, t_{2}\right)} \cong \bigoplus_{\lambda} R_{\lambda}^{(k)}
$$

where the sum is over all $(n, k)$-bipartitions $\lambda=(\mu, \nu)$. such that

$$
\sum_{i \geq k \text { and } \nu_{i}>\nu_{i+1}}(n-i)=t_{1}, \quad \sum_{i<k \text { and } \mu_{i}>\mu_{i+1}} i=t_{2} .
$$

For example, suppose that $k=3$ and $n=4$. Then if $\lambda=(\mu, \nu)$ is a $(4,3)$ partition, then $\mu \in\{(0,0,0),(1,0,0),(1,1,0),(2,1,0)\}$ and $\nu \in\{(0,0),(1,0)\}$. Table 1 lists all the possible (4,3)-partitions. Then for each $\lambda=(\mu, \nu)$, we list the corresponding weight $q^{t_{1}} t^{t_{2}}$ given by (12), the corresponding descent set $A_{\lambda}$, and the ribbon Schur function corresponding to $A_{\lambda}$. 


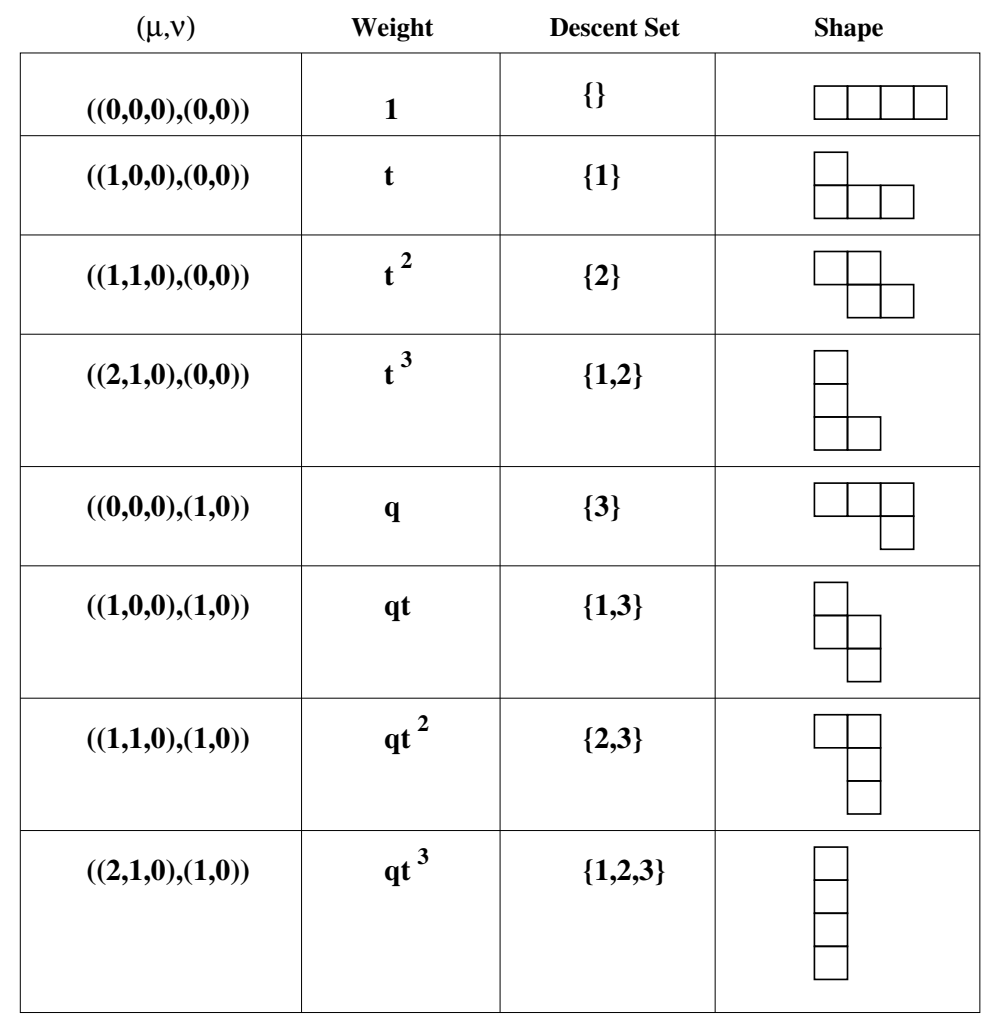

Figure 4: Table 1.

\subsubsection{Decomposition into Irreducibles}

A classical theorem of Lusztig and Stanley gives the multiplicity of the irreducibles in the homogeneous component of the coinvariant algebra of type $A$. Define $1 \leq i<n$ to be a descent in a standard Young tableau $T$ if $i+1$ lies strictly above and weakly to the left of $i$ (in French notation). Denote the set of all descents in $T$ by $\operatorname{Des}(T)$ and let the major index of $T$ be

$$
\operatorname{maj}(T):=\sum_{i \in \operatorname{Des}(T)} i .
$$

Theorem 1.15. [36, Prop. 4.11] The multiplicity of the irreducible $S_{n}$-representation $S^{\lambda}$ in the $k$-th homogeneous component of the coinvariant algebra of type $A$ is

$$
\#\{T \in S Y T(\lambda): \operatorname{maj}(T)=k\}
$$

where $S Y T(\lambda)$ is the set of all standard Young tableaux of shape $\lambda$.

In 1994, Stembridge [38] gave an explicit combinatorial interpretation of the $(q, t)$ Kostka polynomials for hook shape. Stembridge's result implies the following extension of the Lusztig-Stanley theorem. 
For a standard Young tableau $T$ define

$$
\operatorname{maj}_{i, j}(T):=\sum_{\substack{r \in \operatorname{Des}(T) \\ i \leq r<j}} r
$$

and

$$
\operatorname{comaj}_{i, j}(T):=\sum_{\substack{r \in \operatorname{Des}(T) \\ i \leq r<j}}(n-r) .
$$

For example, for the column strict tableaux $T$ pictured in Figure 5 and $k=4, \operatorname{Des}(T)=$ $\{2,3,5,7\}$, maj $_{1,4}(T)=2+3=5$, and comaj $_{5,8}=(8-5)+(8-7)=4$.

\begin{tabular}{|l|l|l|l|}
\hline 4 & 8 & \multicolumn{2}{|c}{} \\
\cline { 1 - 2 } 3 & 6 & \multicolumn{2}{|c}{} \\
\hline 1 & 2 & 5 & 7 \\
\hline
\end{tabular}

Figure 5: Des, maj $_{1, k}$, and comaj $_{k, n}$ for a standard tableau.

We can restate Stembridge's results [38] as follows.

\section{Theorem 1.16.}

$$
\tilde{K}_{\lambda,\left(1^{k-1}, n-k+1\right)}(q, t)=\sum_{T \in S Y T(\lambda)} q^{\mathrm{maj}_{1, n-k+1}(T)} t^{\mathrm{comaj}_{n-k+1, n}(T)} .
$$

Our next result is an immediate consequence of Haiman's result (8) and Theorem 1.16.

Theorem 1.17. The multiplicity of the irreducible $S_{n}$-representation $S^{\lambda}$ in the $\left(h, h^{\prime}\right)$ level of $\mathbf{H}_{\left(1^{k-1}, n-k+1\right)}$ (bi-graded by total degrees in the $x$ 's and $y$ 's) is

$$
\chi_{\lambda}^{\left(h, h^{\prime}\right)}=\#\left\{T \in S Y T(\lambda): \operatorname{maj}_{1, n-k+1}(T)=h, \operatorname{comaj}_{n-k+1, n}(T)=h^{\prime}\right\},
$$

where $S Y T(\lambda)$ is the set of all standard Young tableaux of shape $\lambda$.

Haglund [19] gave another proof of Theorem 1.17 that used his conjectured combinatorial definition of $\tilde{H}_{\mu}(\bar{x} ; q, t)$. Haglund's conjecture has recently been proved by Haglund, Haiman and Loehr [20, 21].

We give two proofs of this decomposition rule. The first one, given in Section 6 , follows from the decomposition into descent representations described in Corollary 1.14 above.

The second proof, given in Section 7, is more "combinatorial". It uses the mechanism of [21] but does not rely on Haglund's combinatorial interpretation of $\tilde{H}_{\left(1^{k-1}, n-k+1\right)}(\bar{x} ; q, t)$. 


\section{The Garsia-Haiman Module $\mathbf{H}_{\mu}$}

In this section, we shall provide some background on the Garsia-Haiman module, also known as the space of orbit harmonics. Proofs of the results in this section can be found in [13] and [14].

Let $m:=2 n$ and $\left(z_{1}, \ldots, z_{m}\right):=\left(x_{1}, \ldots, x_{n}, y_{1}, \ldots, y_{n}\right)$. Given positive weights $w_{1}, \ldots, w_{m}$, define a grading of $\mathbf{R}=\mathbf{Q}\left[z_{1}, \ldots, z_{m}\right]$ by setting, for any monomial $\bar{z}^{p}=$ $z_{1}^{p_{1}} \cdots z_{m}^{p_{m}}$

$$
d_{w}\left(\bar{z}^{p}\right):=\sum_{i=1}^{m} w_{i} p_{i} .
$$

For any polynomial $g=\sum_{p} c_{p} \bar{z}^{p}$ and any $k$, set

$$
\pi_{k}^{w} g=\sum_{d_{w}\left(\bar{z}^{p}\right)=k} c_{p} \bar{z}^{p}
$$

Call $\pi_{k}^{w} g$ the $w$-homogeneous component of $w$-degree $k$, and let top ${ }_{w}(g)$ denote the $w$ homogeneous component of highest $w$-degree in $g$. Let $V$ be a subspace of $\mathbf{R}$. We say that $V$ is $w$-homogeneous if $\pi_{k}^{w} V \subseteq V$ for all $k$. Let $V^{\perp}$ be the orthogonal complement of $V$ with respect to the inner product defined at the beginning of Subsection 1.3. If $\mathrm{V}$ is $w$-homogeneous, then $V^{\perp}$ is also $w$-homogeneous.

If $J$ is an ideal of $\mathbf{R}$ then

$$
J^{\perp}=\left\{g \in \mathbf{Q}[\bar{z}]: f\left(\frac{\partial}{\partial z_{1}}, \ldots, \frac{\partial}{\partial z_{m}}\right) g(\bar{z})=0(\forall f \in J)\right\} .
$$

In particular, $J^{\perp}$ is closed under differentiation. We set $\mathbf{R}_{J}=\mathbf{R} / J$ and define the associated $w$-graded ideal of $J$ to be the ideal

$$
g r_{w} J:=\left\langle\operatorname{top}_{w}(g): g \in J\right\rangle .
$$

The quotient ring $\mathbf{R} / g r_{w} J$ is referred to as the $w$-graded version of $\mathbf{R}_{J}$ and is denoted by $g r_{w} \mathbf{R}_{J}$.

Given a point $\rho=\left(\rho_{1}, \ldots, \rho_{m}\right)$, denote its $S_{n}$-orbit by

$$
[\rho]=[\rho]_{S_{n}}=\left\{\sigma(\rho): \sigma \in S_{n}\right\} .
$$

We also set

$$
\mathbf{R}_{[\rho]}:=\mathbf{R} / J_{[\rho]}
$$

where

$$
J_{[\rho]}=\{g \in \mathbf{R}: g(\bar{z})=0(\forall \bar{z} \in[\rho])\}
$$

Considered as an algebraic variety, $\mathbf{R}_{[\rho]}$ is the coordinate ring of $[\rho]$. Since the ideal $J_{[\rho]}$ is $S_{n}$-invariant, $S_{n}$ also acts on $\mathbf{R}_{[\rho]}$. In fact, it is easy to see that the corresponding representation is equivalent to the action of $S_{n}$ on the left cosets of the stabilizer of $\rho$. Thus if the stabilizer of $\rho$ is trivial, i.e. if $\rho$ is a regular point, then $\mathbf{R}_{[\rho]}$ is a version of 
the left regular representation of $S_{n}$. If each $\sigma \in S_{n}$ preserves $w$-degrees, then we can associate two further $S_{n}$-modules with $\rho$; namely, $g r_{w} J_{[\rho]}$ and its orthogonal complement

$$
\mathbf{H}_{[\rho]}=\left(g r_{w} J_{[\rho]}\right)^{\perp} .
$$

Garsia and Haiman refer to $\mathbf{H}_{[\rho]}$ as the space of harmonics of the orbit $[\rho]$. Clearly if $f(\bar{z})$ is an $S_{n}$-invariant polynomial, then $f(\bar{z})-f(\rho) \in J_{[\rho]}$. In addition, if $f(\bar{z})$ is $w$ homogeneous, then $f(\bar{z}) \in g r_{w} J_{[\rho]}$. This implies that any element $g \in \mathbf{H}_{[\rho]}$ must satisfy the differential equation

$$
f\left(\frac{\partial}{\partial z_{1}}, \ldots, \frac{\partial}{\partial z_{m}}\right) g\left(z_{1}, \ldots, z_{m}\right)=0 .
$$

It is not difficult to show that $\mathbf{H}_{[\rho]}$ and $g r_{w} \mathbf{R}_{[\rho]}$ are equivalent $w$-graded modules and that these two spaces realize a graded version of the regular representations of $S_{n}$.

Given a partition $\mu=\left(\mu_{1} \geq \cdots \geq \mu_{k}>0\right)$ with $k$ parts, let $h=\mu_{k}$ be the number of parts of the conjugate partition $\mu^{\prime}$. Let $\alpha_{1}, \ldots, \alpha_{k}$ and $\beta_{1}, \ldots, \beta_{h}$ be distinct rational numbers. Alternatively, we can think of the $\alpha$ 's and $\beta$ 's as indeterminates. An injective tableau of shape $\mu$ is a labelling of the cells of $\mu$ by the numbers $\{1,2, \ldots, n\}$ so that no two cells are labelled by the same number. The collection of all such tableaux will be denoted by $\mathcal{I} T_{\mu}$. For each $T \in \mathcal{I} T_{\mu}$ and $\ell \in\{1,2, \ldots, n\}$, let $s_{T}(\ell)=\left(i_{T}(\ell), j_{T}(\ell)\right)$ denote the cell of $\mu$ which contains the number $\ell$. We then construct a point $\rho(T)=(a(T), b(T))$ in $2 n$-dimensional space by setting

$$
a_{\ell}(T):=\alpha_{i_{T}(\ell)} \text { and } b_{\ell}(T)=\beta_{j_{T}(\ell)} \quad(1 \leq \ell \leq n) .
$$

For example, for the injective tableau $T$ pictured in Figure 6,

$$
\begin{aligned}
& \rho(T)=\left(\alpha_{2}, \alpha_{1}, \alpha_{1}, \alpha_{1}, \alpha_{3}, \alpha_{2}, \alpha_{2}, \alpha_{1}, \alpha_{4} ; \beta_{2}, \beta_{1}, \beta_{3}, \beta_{2}, \beta_{1}, \beta_{3}, \beta_{1}, \beta_{4}, \beta_{1}\right)
\end{aligned}
$$

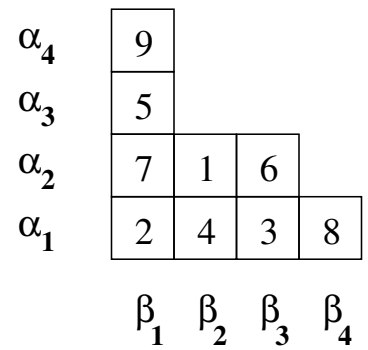

Figure 6: $\rho(T)$.

The collection $\left\{\rho(T): T \in \mathcal{I} T_{\mu}\right\}$ consists of $n$ ! points. In fact, it is simply the $S_{n}$-orbit under the diagonal action of any one of its elements, where the diagonal action of $S_{n}$ on $2 n$-dimensional space is defined by

$$
\sigma\left(x_{1}, \ldots, x_{n} ; y_{1}, \ldots, y_{n}\right):=\left(x_{\sigma_{1}}, \ldots, x_{\sigma_{n}} ; y_{\sigma_{1}}, \ldots, y_{\sigma_{n}}\right)
$$


for any $\sigma \in S_{n}$. For the rest of this paper we let

$$
\mathbf{R}=\mathbf{Q}[\bar{x}, \bar{y}]=\mathbf{Q}\left[x_{1}, \ldots, x_{n} ; y_{1}, \ldots, y_{n}\right]
$$

and use the grading $w_{i}=1$ for all $i=1, \ldots, 2 n$. We shall let $\mathbf{R}_{\left[\rho_{\mu}\right]}, g r \mathbf{R}_{\left[\rho_{\mu}\right]}$, and $\mathbf{H}_{\left[\rho_{\mu}\right]}$ denote the corresponding coordinate ring, its graded version, and the space of harmonics for the $S_{n}$-orbit $\left\{\rho(T): T \in \mathcal{I} T_{\mu}\right\}$.

Garsia and Haiman [13] proved the following.

Proposition 2.1. If $(i, j)$ is a cell outside of $\mu$, then for any $s \in\{1, \ldots, n\}$, the monomial $x_{s}^{i-1} y_{s}^{j-1}$ belongs to the ideal gr $J_{\left[\rho_{\mu}\right]}$. In particular, if $x^{p} y^{q}=x_{1}^{p_{1}} \cdots x_{n}^{p_{n}} y_{1}^{q_{1}} \cdots y_{n}^{q_{n}} \notin J_{\left[\rho_{\mu}\right]}$, then all the pairs $\left(p_{s}, q_{s}\right)$ must be cells of $\mu$.

Theorem 2.2. Let $\mathbf{H}_{\mu}$ be the vector space of polynomials spanned by all the partial derivatives of $\Delta_{\mu}\left(x_{1}, \ldots, x_{n} ; y_{1}, \ldots, y_{n}\right)$ (see (4) above). Then

1. $\mathbf{H}_{\mu} \subseteq \mathbf{H}_{\left[\rho_{\mu}\right]}$.

2. If $\operatorname{dim}\left(\mathbf{H}_{\mu}\right)=n$ !, then $\mathbf{H}_{\mu}=\mathbf{H}_{\left[\rho_{\mu}\right]}$ and $J_{\mu}=\mathbf{H}_{\mu}^{\perp}=\operatorname{gr} J_{\left[\rho_{\mu}\right]}$.

In 2001, Haiman solved the $n$ ! conjecture and proved that the dimension of $\mathbf{H}_{\mu}$ is indeed $n$ ! [23]. Thus, Theorem 2.2 implies that for every partition $\mu, J_{\mu}=\mathbf{H}_{\mu}^{\perp}$ is the ideal in $\mathbf{R}$ generated by the set

$$
g r\left\{f \in \mathbf{R}: f(\rho(T))=0 \quad\left(\forall T \in \mathcal{I} T_{\mu}\right)\right\} .
$$

We deduce

Corollary 2.3. The following polynomials belong to the ideal $J_{\left(1^{k-1}, n-k+1\right)}$ :

(i) $\Lambda\left(x_{1}, \ldots, x_{n}\right)^{+}$and $\Lambda\left(y_{1}, \ldots, y_{n}\right)^{+}$(the symmetric functions in $x$ and $y$ without a constant term),

(ii) the monomials $x_{i_{1}} \cdots x_{i_{k}}\left(i_{1}<\cdots<i_{k}\right)$ and $y_{i_{1}} \cdots y_{i_{n-k+1}}\left(i_{1}<\cdots<i_{n-k+1}\right)$, and

(iii) the monomials $x_{i} y_{i}(1 \leq i \leq n)$.

\section{Proof.}

(i) The multiset of row-coordinates is the same for all injective tableaux of a fixed shape. Thus, for any $f \in \Lambda\left(x_{1}, \ldots, x_{n}\right)^{+}, g(\bar{z}):=f\left(x_{1}, \ldots, x_{n}\right)-f\left(\alpha_{1}, \ldots, \alpha_{1}, \alpha_{2}, \ldots, \alpha_{k}\right)$ is zero for all substitutions $\bar{z}=\rho(T)$, where $T$ is an injective tableau of shape $(n-$ $\left.k+1,1^{k-1}\right)$. Thus $f=\operatorname{top}(g) \in J_{\left(n-k+1,1^{k-1}\right)}$. Similarly, for any $f \in \Lambda\left(y_{1}, \ldots, y_{n}\right)^{+}$, $g(\bar{z}):=f\left(y_{1}, \ldots, y_{n}\right)-f\left(\beta_{1}, \ldots, \beta_{1}, \beta_{2}, \ldots, \beta_{n-k+1}\right)$ is zero for all substitutions $\bar{z}=$ $\rho(T)$. Thus $f=\operatorname{top}(g) \in J_{\left(n-k+1,1^{k-1}\right)}$. 
(ii) In the shape $\left(1^{k-1}, n-k+1\right)$ there are only $k-1$ cells outside of row 1 . Therefore $g(\bar{z}):=\left(x_{i_{1}}-\alpha_{1}\right) \cdots\left(x_{i_{k}}-\alpha_{1}\right)$ is zero for all $1 \leq i_{1}<\cdots<i_{k} \leq n$ and all $\bar{z}=\rho(T)$. Thus $x_{i_{1}} \cdots x_{i_{k}}=\operatorname{top}(g) \in J_{\left(n-k+1,1^{k-1}\right)}$. Similarly, $g(\bar{z}):=\left(y_{i_{1}}-\beta_{1}\right) \cdots\left(y_{i_{n-k+1}}-\beta_{1}\right)$ is zero for all $1 \leq i_{1}<\cdots<i_{n-k+1} \leq n$ and all $\bar{z}=\rho(T)$. Thus $y_{i_{1}} \cdots y_{i_{n-k+1}}=$ $\operatorname{top}(g) \in J_{\left(n-k+1,1^{k-1}\right)}$.

(iii) Each cell of a hook shape is either in row 1 or in column 1 . Thus $g(\bar{z}):=\left(x_{i}-\alpha_{1}\right)\left(y_{i}-\right.$ $\left.\beta_{1}\right)$ is zero for all $1 \leq i \leq n$ and all $\bar{z}=\rho(T)$, so that $x_{i} y_{i}=\operatorname{top}(g) \in J_{\left(n-k+1,1^{k-1}\right)}$.

In the sequel we will show that the polynomials in Corollary 2.3 actually generate the ideal $J_{\left(1^{k-1}, n-k+1\right)}$.

\section{Generalized Kicking-Filtration Process}

In this section we prove Theorem 1.5, which implies Corollary 1.7 as a special case and Theorem 1.4. The idea is to generalize the kicking process for obtaining a basis. The kicking process was used in an early paper of Garsia and Haiman [13] to prove the $n$ !conjecture for hooks. We combine ingredients of this process with a filtration.

\subsection{Proof of Theorem 1.5}

For every triple $(A, c, \bar{A})$, where $[n]=A \cup\{c\} \cup \bar{A}$ and $|A|=k-1,|\bar{A}|=n-k$, define an $(A, c, \bar{A})$-permutation $\pi_{(A, c, \bar{A})} \in S_{n}$, in which the letters of $A$ appear in decreasing order, then $c$, and then the remaining letters in increasing order. For example, if $n=9, k=$ $4, c=5, A=\{1,6,7\}$ then $\pi_{(A, c, \bar{A})}=761523489$.

For given $n$ and $k$, order the $N:=n\left(\begin{array}{l}n-1 \\ k-1\end{array}\right)$ distinct $(A, c, \bar{A})$-permutations in reverse lexicographic order (as words): $\pi_{1}, \ldots, \pi_{N}$. If $\pi_{i}$ corresponds to $(A, c, \bar{A})$, let $m_{i}:=m_{(A, c, \bar{A})}$ (see Subsection 1.3.2 above).

For example, for $n=4$ and $k=3$, the list of $(A, c, \bar{A})$-permutations is

$$
\begin{gathered}
\pi_{(\{34\}, 2,\{1\})}=4321, \pi_{(\{34\}, 1,\{2\})}=4312, \pi_{(\{24\}, 3,\{1\})}=4231, \ldots, \\
\pi_{(\{13\}, 2,\{4\})}=3124, \pi_{(\{12\}, 4,\{3\})}=2143, \pi_{(\{12\}, 3,\{4\})}=2134 .
\end{gathered}
$$

and the order is

$$
\begin{gathered}
4321<_{L} 4312<_{L} 4231<_{L} 4213<_{L} 4132 \\
<_{L} 4123<_{L} 3241<_{L} 3214<_{L} 3142<_{L} 3124<_{L} 2143<_{L} 2134 .
\end{gathered}
$$

Thus the permutations are indexed by $\pi_{1}=4321, \pi_{2}=4312, \pi_{3}=4231, \ldots, \pi_{11}=$ 2143, $\pi_{N}=\pi_{12}=2134$ and the corresponding monomials are $m_{1}=x_{4} x_{3} y_{1}, m_{2}=$ $x_{4} x_{3}, m_{3}=x_{4} y_{1}, \ldots, m_{11}=y_{3}, m_{N}=m_{12}=1$.

Let

$$
I_{0}:=J_{\left(1^{k-1}, n-k+1\right)}
$$


and define recursively

$$
I_{t}:=I_{t-1}+m_{t} \mathbf{Q}[\bar{x}, \bar{y}] \quad(1 \leq t \leq N) .
$$

Clearly,

$$
I_{0} \subseteq I_{1} \subseteq I_{2} \subseteq \cdots \subseteq I_{N}=\mathbf{Q}[\bar{x}, \bar{y}]
$$

The last equality follows from the fact that $m_{N}=1$.

Observation 3.1.

$$
\mathbf{H}_{\left(1^{k-1}, n-k+1\right)}^{\prime}=\mathbf{Q}[\bar{x}, \bar{y}] / I_{0} \cong \bigoplus_{t=1}^{N}\left(I_{t} / I_{t-1}\right)
$$

as vector spaces. In particular, a sequence of bases for the quotients $I_{t} / I_{t-1}, 1 \leq t \leq N$, will give a basis for $\mathbf{H}_{\left(1^{k-1}, n-k+1\right)}^{\prime}$.

It remains to prove that the set $m_{t} \cdot B_{A} \cdot C_{\bar{A}}$, where $B_{A}, C_{\bar{A}}$ are bases of coinvariant algebras in $\bar{x}_{A}$ and $\bar{y}_{\bar{A}}$ respectively, is a basis for $I_{t} / I_{t-1}$.

Consider the natural projection

$$
f_{t}: m_{t} \mathbf{Q}[\bar{x}, \bar{y}] \longrightarrow I_{t} / I_{t-1}
$$

Clearly, $f_{t}$ is a surjective map.

\section{Lemma 3.2.}

$$
m_{t} \cdot\left(\sum_{i \notin A}\left\langle x_{i}\right\rangle+\sum_{j \notin \bar{A}}\left\langle y_{j}\right\rangle+\left\langle\Lambda[\bar{x}]^{+}\right\rangle+\left\langle\Lambda[\bar{y}]^{+}\right\rangle\right) \subseteq I_{t-1} \cap m_{t} \mathbf{Q}[\bar{x}, \bar{y}]=\operatorname{ker}\left(f_{t}\right),
$$

so that $f_{t}$ is well defined on the quotient

$$
\begin{gathered}
m_{t} \mathbf{Q}[\bar{x}, \bar{y}] /\left[m_{t} \cdot\left(\sum_{i \notin A}\left\langle x_{i}\right\rangle+\sum_{j \notin \bar{A}}\left\langle y_{j}\right\rangle+\left\langle\Lambda[\bar{x}]^{+}\right\rangle+\left\langle\Lambda[\bar{y}]^{+}\right\rangle\right)\right] \cong \\
m_{t} \cdot \mathbf{Q}\left[\bar{x}_{A}\right] /\left\langle\Lambda\left[\bar{x}_{A}\right]^{+}\right\rangle \cdot \mathbf{Q}\left[\bar{y}_{\bar{A}}\right] /\left\langle\Lambda\left[\bar{y}_{\bar{A}}\right]^{+}\right\rangle .
\end{gathered}
$$

Proof of Lemma 3.2. First, let $i \notin A$. We shall show that $m_{t} x_{i} \in I_{t-1}$. Consider the four possible cases $\mathrm{X} 1-\mathrm{X} 4$ :

X1. $a>c$ for all $a \in A$.

Then $m_{t}$ has all the $x_{a}(a \in A)$ as factors, so that $m_{t} x_{i}$ has exactly $k$ distinct $x$-factors and therefore belongs to $I_{0} \subseteq I_{t-1}$ (by Corollary 2.3(ii)).

X2. $i<c$ and there exists $a \in A$ such that $a<c$.

$i \notin A$ and $i \neq c$, hence $i \in \bar{A}$. As $i<c, y_{i}$ is a factor of $m_{t}$. Hence $x_{i} y_{i}$ is a factor of $m_{t} x_{i}$ and therefore belongs to $I_{0} \subseteq I_{t-1}$ (by Corollary 2.3(iii)). 
X3. $i=c$ and there exists $a \in A$ such that $a<c$.

Let $a_{0}:=\max \{a \in A: a<c\}$. Let $t_{\left(c, a_{0}\right)}$ be the transposition interchanging $c$ with $a_{0}$ and let $A^{\prime}:=\left(A \backslash\left\{a_{0}\right\}\right) \cup\{c\}$. By the definition of $a_{0}, m_{t^{\prime}}=m_{\left(A^{\prime}, a_{0}, \bar{A}^{\prime}\right)}$ divides $m_{t} x_{i}$. To verify this, notice that for every $j$, such that $x_{j}$ divides $m_{t^{\prime}}, j \in A^{\prime}$ and $j>a_{0}$. Hence $j \neq a_{0}$ and thus $j \in A$. Also, by the definition of $a_{0}$, for every $j \in A$, $j>a_{0} \Rightarrow j>c$. It follows that $x_{j}$ divides $m_{t}$. Finally, for every $j$, such $y_{j}$ divides $m_{t^{\prime}}, j \in \bar{A}=\bar{A}^{\prime}$ and $j<a_{0}$. Hence $j<c$ and $y_{j}$ divides $m_{t}$.

On the other hand, $\pi_{\left(A^{\prime}, a_{0}, \bar{A}^{\prime}\right)}=t_{\left(c, a_{0}\right)} \pi_{(A, c, \bar{A})}<_{L} \pi_{(A, c, \bar{A})}$, since $a_{0}<c$. Hence $m_{t^{\prime}}=m_{\left(A^{\prime}, a_{0}, \bar{A}^{\prime}\right)} \in I_{t-1}$ and $m_{t} x_{i} \in\left\langle m_{t^{\prime}}\right\rangle \subseteq I_{t-1}$.

X4. $i>c$ and there exists $a \in A$ such that $a<c$.

Let $a_{0}:=\max \{a \in A: a<c\}$ as above. Let $t_{\left(a_{0}, i, c\right)}$ be the 3-cycle mapping $a_{0}$ to $i, i$ to $c$ and $c$ to $a_{0}$. Let $A^{\prime}:=\left(A \backslash\left\{a_{0}\right\}\right) \cup\{i\}$ and $m_{t^{\prime}}=m_{\left(A^{\prime}, a_{0}, \bar{A}^{\prime}\right)}$. Then $\pi_{\left(A^{\prime}, a_{0}, \bar{A}^{\prime}\right)} \leq_{L} t_{\left(a_{0}, i, c\right)} \pi_{(A, c, \bar{A})}<_{L} \pi_{(A, c, \bar{A})}$, since $a_{0}<c<i$. Thus $t^{\prime}<t$.

On the other hand, $m_{t^{\prime}}=m_{\left(A^{\prime}, a_{0}, \bar{A}^{\prime}\right)}$ divides $m_{t} x_{i}$. This follows from the implications below:

$$
i^{\prime} \in A^{\prime} \text { and } i^{\prime}>a_{0} \Longrightarrow i^{\prime}=i \text { or } i^{\prime}>c .
$$

Hence, every $x_{i^{\prime}}$ which divides $m_{t^{\prime}}$ divides also $m_{t} x_{i}$. Also

$$
j^{\prime} \in \bar{A}^{\prime} \text { and } j^{\prime}<a_{0} \Longrightarrow j^{\prime} \in \bar{A} \text { and } j^{\prime}<c .
$$

Hence, every $y_{j^{\prime}}$ which divides $m_{t^{\prime}}$ divides also $m_{t} x_{i}$.

We conclude that $m_{t^{\prime}}=m_{\left(A^{\prime}, a_{0}, \bar{A}\right)} \in I_{t-1}$ and $\left\langle m_{t} x_{i}\right\rangle \subseteq\left\langle m_{\left(A^{\prime}, a_{0}, \bar{A}\right)}\right\rangle \subseteq I_{t-1}$.

Similarly, by considering four analogous cases, one can show that if $j \notin \bar{A}$ then $m_{t} y_{j} \in$ $I_{t-1}$.

Finally, observe that, by Corollary 2.3(i), $m_{t}\left\langle\Lambda[\bar{x}]^{+}\right\rangle \subseteq I_{0} \subseteq I_{t-1}$ and $m_{t}\left\langle\Lambda[\bar{y}]^{+}\right\rangle \subseteq$ $I_{0} \subseteq I_{t-1}$.

This completes the proof of Lemma 3.2.

Now, recall that $B_{A}$ and $C_{\bar{A}}$ are bases for the coinvariant algebras $\mathbf{Q}\left[\bar{x}_{A}\right] /\left\langle\Lambda\left[\bar{x}_{A}\right]^{+}\right\rangle$and $\mathbf{Q}\left[\bar{y}_{\bar{A}}\right] /\left\langle\Lambda\left[\bar{y}_{\bar{A}}\right]^{+}\right\rangle$respectively, so that $m_{t} \cdot B_{A} \cdot C_{\bar{A}}$ spans $m_{t} \cdot \mathbf{Q}\left[\bar{x}_{A}\right] /\left\langle\Lambda\left[\bar{x}_{A}\right]^{+}\right\rangle \cdot \mathbf{Q}\left[\bar{y}_{\bar{A}}\right] /\left\langle\Lambda\left[\bar{y}_{\bar{A}}\right]^{+}\right\rangle$.

In order to prove Theorem 1.5, it remains to show that for every $1 \leq t \leq N$,

(a) $m_{t} \cdot B_{A} \cdot C_{\bar{A}}$ is a basis for $m_{t} \cdot \mathbf{Q}\left[\bar{x}_{A}\right] /\left\langle\Lambda\left[\bar{x}_{A}\right]^{+}\right\rangle \cdot \mathbf{Q}\left[\bar{y}_{\bar{A}}\right] /\left\langle\Lambda\left[\bar{y}_{\bar{A}}\right]^{+}\right\rangle$, and

(b) $f_{t}$ is one-to-one.

Indeed, by Lemma 3.2,

$$
\begin{aligned}
\operatorname{dim}\left(I_{t} / I_{t-1}\right) & \leq \operatorname{dim}\left(m_{t} \cdot \mathbf{Q}\left[\bar{x}_{A}\right] /\left\langle\Lambda\left[\bar{x}_{A}\right]^{+}\right\rangle \cdot \mathbf{Q}\left[\bar{y}_{\bar{A}}\right] /\left\langle\Lambda\left[\bar{y}_{\bar{A}}\right]^{+}\right\rangle\right) \\
& \leq \operatorname{dim}\left(\mathbf{Q}\left[\bar{x}_{A}\right] /\left\langle\Lambda\left[\bar{x}_{A}\right]^{+}\right\rangle \mathbf{Q}\left[\bar{y}_{\bar{A}}\right] /\left\langle\Lambda\left[\bar{y}_{\bar{A}}\right]^{+}\right\rangle\right)=(k-1) ! \cdot(n-k) !
\end{aligned}
$$


The last equality follows from the classical result that the coinvariant algebra of $S_{n}$ carries the regular $S_{n}$-representation $[24, \S 3]$.

If there exists $1 \leq t \leq N$ such that either (a) or (b) does not hold, then there exists $t$ for which a sharp inequality holds in (23). Then

$$
\begin{gathered}
\operatorname{dim} \mathbf{H}_{\left(1^{k-1}, n-k+1\right)}^{\prime}=\operatorname{dim}\left(\mathbf{Q}[\bar{x}, \bar{y}] / I_{0}\right)=\sum_{t=1}^{N} \operatorname{dim}\left(I_{t} / I_{t-1}\right) \\
<N(k-1) !(n-k) !=n\left(\begin{array}{c}
n-1 \\
k-1
\end{array}\right)(k-1) !(n-k) !=n !,
\end{gathered}
$$

contradicting the $n$ ! theorem. This completes the proof of Theorem 1.5.

\subsection{Applications}

Proof of Corollary 1.7. By choosing $B_{A}$ and $C_{\bar{A}}$ in Theorem 1.5 as Artin bases of the corresponding coinvariant algebras we get the $k$-th Artin basis $\left(b_{\pi}^{(k)}\right)$.

By choosing $B_{A}$ and $C_{\bar{A}}$ as descent bases we get the $k$-th descent basis $\left(a_{\pi}^{(k)}\right)$.

Finally, by choosing $B_{A}$ as a descent basis and $C_{\bar{A}}$ as an Artin basis we get the $k$-th Haglund basis $\left(c_{\pi}^{(k)}\right)$.

Proof of Theorem 1.4. Let $J^{\prime}$ be the ideal of $\mathbf{Q}[\bar{x}, \bar{y}]$ generated by $\Lambda\left(x_{1}, \ldots, x_{n}\right)^{+}$, $\Lambda\left(y_{1}, \ldots, y_{n}\right)^{+}$and the monomials $x_{i_{1}} \cdots x_{i_{k}}\left(i_{1}<\cdots<i_{k}\right), y_{i_{1}} \cdots y_{i_{n-k+1}}\left(i_{1}<\cdots<\right.$ $\left.i_{n-k+1}\right)$, and $x_{i} y_{i}(1 \leq i \leq n)$. By Corollary 2.3, $J^{\prime} \subseteq J_{\left(1^{k-1}, n-k+1\right)}$.

In the above proof of Theorem 1.5 , if one replaces $I_{0}:=J_{\left(1^{k-1}, n-k+1\right)}$ by $I_{0}:=J^{\prime}$, the same conclusions hold:

$$
\operatorname{dim}\left(\mathbf{Q}[\bar{x}, \bar{y}] / J^{\prime}\right)=\sum_{t=1}^{N} \operatorname{dim}\left(I_{t} / I_{t-1}\right) \leq N(k-1) !(n-k) !=n !
$$

On the other hand, by [13],

$$
\operatorname{dim}\left(\mathbf{Q}[\bar{x}, \bar{y}] / J^{\prime}\right) \geq \operatorname{dim}\left(\mathbf{Q}[\bar{x}, \bar{y}] / J_{\left(1^{k-1}, n-k+1\right)}\right)=\operatorname{dim} \mathbf{H}_{\left(1^{k-1}, n-k+1\right)}^{\prime}=n ! .
$$

Thus equality holds everywhere, and $J^{\prime}=J_{\left(1^{k-1}, n-k+1\right)}$.

\section{A $k$-th Analogue of the Polynomial Ring}

The current section provides an appropriate setting for an extension of the straightening algorithm from the coinvariant algebra $[4,1]$ to the Garsia-Haiman hook modules. The algorithm will be given in Section 5 and will be used later to describe the $S_{n}$-action on $\mathbf{H}_{\left(1^{k-1}, n-k+1\right)}^{\prime}$ and resulting decomposition rules (see Section 6). 


\section{1 $\mathcal{P}_{n}^{(k)}$ and its Monomial Basis}

Definition 4.1. For every $1 \leq k \leq n$ let $\mathcal{I}_{k}$ be the ideal in $\mathbf{Q}\left[x_{1}, \ldots, x_{n}, y_{1}, \ldots, y_{n}\right]$ generated by

(i) the monomials $x_{i_{1}} \cdots x_{i_{k}}\left(1 \leq i_{1}<\cdots<i_{k} \leq n\right)$,

(ii) the monomials $y_{i_{1}} \cdots y_{i_{n-k+1}}\left(1 \leq i_{1}<\cdots<i_{n-k+1} \leq n\right)$, and

(iii) the monomials $x_{i} y_{i}(1 \leq i \leq n)$.

Denote

$$
\mathcal{P}_{n}^{(k)}:=\mathbf{Q}\left[x_{1}, \ldots, x_{n}, y_{1}, \ldots, y_{n}\right] / \mathcal{I}_{k} .
$$

Claim 4.2. The ideal $\mathcal{I}_{k}$ is contained in the ideal $J_{\left(1^{k-1}, n-k+1\right)}$.

Proof. Immediate from Definition 4.1 and Corollary 2.3.

Definition 4.3. For a monomial $m=\prod_{i=1}^{n} x_{i}^{p_{i}} \prod_{j=1}^{n} y_{j}^{q_{j}} \in \mathbf{Q}\left[x_{1}, \ldots, x_{n}, y_{1}, \ldots, y_{n}\right]$ define the $x$-support and the $y$-support

$$
\operatorname{Supp}_{x}(m):=\left\{i: p_{i}>0\right\}, \quad \operatorname{Supp}_{y}(m):=\left\{j: q_{j}>0\right\}
$$

Definition 4.4. Let $M_{n}^{(k)}$ be the set of all monomials in $\mathbf{Q}\left[x_{1}, \ldots, x_{n}, y_{1}, \ldots, y_{n}\right]$ with

(i) $\left|\operatorname{Supp}_{x}(m)\right| \leq k-1$,

(ii) $\left|\operatorname{Supp}_{y}(m)\right| \leq n-k$, and

(iii) $\operatorname{Supp}_{x}(m) \cap \operatorname{Supp}_{y}(m)=\emptyset$.

Observation 4.5. $\left\{m+\mathcal{I}_{k}: m \in M_{n}^{(k)}\right\}$ is a basis for $\mathcal{P}_{n}^{(k)}$.

Example 4.6. Let $k=n$. Then

$$
\mathcal{P}_{n}^{(n)} \cong \mathbf{Q}\left[x_{1}, \ldots, x_{n}\right] /\left\langle x_{1} \cdots x_{n}\right\rangle
$$

and $M_{n}^{(n)}$ consists of all the monomials in $\mathbf{Q}\left[x_{1}, \ldots, x_{n}\right]$ which do not involve all the $x$ variables, i.e., are not divisible by $x_{1} \cdots x_{n}$. Similarly,

$$
\mathcal{P}_{n}^{(1)} \cong \mathbf{Q}\left[y_{1}, \ldots, y_{n}\right] /\left\langle y_{1} \cdots y_{n}\right\rangle
$$

and $M_{n}^{(1)}$ consists of all the monomials in $\mathbf{Q}\left[y_{1}, \ldots, y_{n}\right]$ which do not involve all the $y$ variables. 


\subsection{A Bijection}

Next, define a map $\psi^{(k)}: M_{n}^{(k)} \rightarrow M_{n}^{(n)}$.

Every monomial $m \in M_{n}^{(k)}$ has the form $m=x_{i_{1}}^{p_{i_{1}}} \cdots x_{i_{k-1}}^{p_{i_{k-1}}} \cdot y_{j_{1}}^{q_{j_{1}}} \cdots y_{j_{n-k}}^{q_{j_{n-k}}}$ (with disjoint supports of $x$ 's and $y$ 's). Let $u:=\max _{j} q_{j}$ and define

$$
\psi^{(k)}(m):=x_{i_{1}}^{p_{i_{1}}} \cdots x_{i_{k-1}}^{p_{i_{k-1}}} \cdot x_{j_{1}}^{-q_{j_{1}}} \cdots x_{j_{n-k}}^{-q_{j_{n-k}}} \cdot\left(x_{1} \cdots x_{n}\right)^{u} .
$$

Notice that if $u=0$, then $\psi^{(k)}(m)=m \in M_{n}^{(k)} \cap \mathbf{Q}\left[x_{1}, \ldots, x_{n}\right]$ has $\left|\operatorname{Supp}_{x}(m)\right| \leq k-1 \leq$ $n-1$ so that $\psi^{(k)}(m) \in M_{n}^{(n)}$, and, if $u>0$ is attained at $y_{j_{0}}$, then the exponent of $x_{j_{0}}$ in $\psi^{(k)}(m)$ is zero so that again $\psi^{(k)}(m) \in M_{n}^{(n)}$.

Example 4.7. Let $n=8, k=5$, and $m=x_{3}^{6} x_{4}^{6} x_{7}^{5} y_{6}^{2} y_{2}^{3} \in M_{8}^{(5)}$. Then $u=3, j_{0}=2$, and

$$
\psi^{(k)}(m)=x_{3}^{6} x_{4}^{6} x_{7}^{5} x_{6}^{-2} x_{2}^{-3} \cdot\left(x_{1} \cdots x_{8}\right)^{3}=x_{3}^{9} x_{4}^{9} x_{7}^{8} x_{1}^{3} x_{5}^{3} x_{8}^{3} x_{6}^{1} \in M_{8}^{(8)} .
$$

We claim that the map $\psi^{(k)}: M_{n}^{(k)} \rightarrow M_{n}^{(n)}$ is a bijection. This will be proved by defining an inverse map $\phi^{(k)}: M_{n}^{(n)} \rightarrow M_{n}^{(k)}$.

Recall from [1] that the index permutation of a monomial $m=\prod_{i=1}^{n} x_{i}^{p_{i}} \in \mathbf{Q}\left[x_{1}, \ldots, x_{n}\right]$ is the unique permutation $\pi=\pi(m) \in S_{n}$ such that

$$
p_{\pi(i)} \geq p_{\pi(i+1)} \quad(1 \leq i<n)
$$

and

$$
p_{\pi(i)}=p_{\pi(i+1)} \Longrightarrow \pi(i)<\pi(i+1)
$$

In other words, $\pi$ reorders the variables $x_{i}$ by (weakly) decreasing exponents, where the variables with a given exponent are ordered by increasing indices. Also, let $\lambda=\lambda(m)=$ $\left(\lambda_{1}, \ldots, \lambda_{n}\right)$ be the corresponding exponent partition, where $\lambda_{i}$ is the exponent of $x_{\pi(i)}$ in $m$. Finally, let $\lambda^{\prime}=\left(\lambda_{1}^{\prime}, \ldots, \lambda_{\ell}^{\prime}\right)$ be the partition conjugate to $\lambda$.

Every monomial $m=\prod_{i=1}^{n} x_{\pi(i)}^{\lambda_{i}} \in M_{n}^{(n)}$ can thus be written in the form

$$
m=\prod_{t=1}^{\ell}\left(x_{\pi(1)} \cdots x_{\pi\left(\lambda_{t}^{\prime}\right)}\right)
$$

Note that, since $m$ is not divisible by $x_{1} \cdots x_{n}, \lambda_{n}=0$ and therefore $\lambda_{t}^{\prime}<n(\forall t)$. Define

$$
\phi^{(k)}(m):=\prod_{\lambda_{t}^{\prime} \leq k-1}\left(x_{\pi(1)} \cdots x_{\pi\left(\lambda_{t}^{\prime}\right)}\right) \cdot \prod_{\lambda_{t}^{\prime} \geq k}\left(y_{\pi\left(\lambda_{t}^{\prime}+1\right)} \cdots y_{\pi(n)}\right) .
$$

Proposition 4.8. $\psi^{(k)}: M_{n}^{(k)} \rightarrow M_{n}^{(n)}$ and $\phi^{(k)}: M_{n}^{(n)} \rightarrow M_{n}^{(k)}$ are inverse maps. 
Proof. Take a monomial $m=\prod_{i=1}^{n} x_{\pi(i)}^{\lambda_{i}} \in M_{n}^{(n)}$ as above. Then

$$
\begin{aligned}
& \operatorname{Supp}_{x}\left(\phi^{(k)}(m)\right) \subseteq\{\pi(1), \ldots, \pi(k-1)\}, \\
& \operatorname{Supp}_{y}\left(\phi^{(k)}(m)\right) \subseteq\{\pi(k+1), \ldots, \pi(n)\},
\end{aligned}
$$

and therefore $\phi^{(k)}(m) \in M_{n}^{(k)}$.

Now apply $\psi^{(k)}$ to $\phi^{(k)}(m)$. Clearly, the highest exponent of a $y$-variable (say $\left.y_{\pi(n)}\right)$ in $\phi^{(k)}(m)$ is $u=\#\left\{t: \lambda_{t}^{\prime} \geq k\right\}\left(=\lambda_{k}\right)$. Thus

$$
\begin{aligned}
\psi^{(k)}\left(\phi^{(k)}(m)\right) & =\prod_{\lambda_{t}^{\prime} \leq k-1}\left(x_{\pi(1)} \cdots x_{\pi\left(\lambda_{t}^{\prime}\right)}\right) \cdot \prod_{\lambda_{t}^{\prime} \geq k}\left(x_{\pi\left(\lambda_{t}^{\prime}+1\right)} \cdots x_{\pi(n)}\right)^{-1} \cdot\left(x_{1} \cdots x_{n}\right)^{u} \\
& =\prod_{\lambda_{t}^{\prime} \leq k-1}\left(x_{\pi(1)} \cdots x_{\pi\left(\lambda_{t}^{\prime}\right)}\right) \cdot \prod_{\lambda_{t}^{\prime} \geq k}\left(x_{\pi(1)} \cdots x_{\pi\left(\lambda_{t}^{\prime}\right)}\right)=m .
\end{aligned}
$$

Conversely, take $m^{\prime} \in M_{n}^{(k)}$. We want to show that $\phi^{(k)}\left(\psi^{(k)}\left(m^{\prime}\right)\right)=m^{\prime}$. We can write

$$
m^{\prime}=\prod_{i=1}^{k-1} x_{\pi(i)}^{\mu_{i}} \cdot \prod_{i=k+1}^{n} y_{\pi(i)}^{\mu_{i}},
$$

where $\mu_{1} \geq \ldots \geq \mu_{k-1} \geq 0$ and $0 \leq \mu_{k+1} \leq \ldots \leq \mu_{n}$. Here $\pi$ is the unique permutation that orders first the indices $i \in \operatorname{Supp}_{x}\left(m^{\prime}\right)$, then the indices $i \notin \operatorname{Supp}_{x}\left(m^{\prime}\right) \cup \operatorname{Supp}_{y}\left(m^{\prime}\right)$, and then the indices $i \in \operatorname{Supp}_{y}\left(m^{\prime}\right)$. The $x$-indices are ordered by weakly decreasing exponents, the $y$-indices are ordered by weakly increasing exponents, and indices with equal exponents are ordered in increasing (index) order. The variables with a given exponent are ordered by increasing indices. The highest exponent of a $y$-variable in $m^{\prime}$ is $u=\mu_{n}$, and therefore

$$
\psi^{(k)}\left(m^{\prime}\right)=\prod_{i=1}^{k-1} x_{\pi(i)}^{u+\mu_{i}} \cdot x_{\pi(k)}^{u} \cdot \prod_{i=k+1}^{n} x_{\pi(i)}^{u-\mu_{i}}=\prod_{i=1}^{n} x_{\pi(i)}^{\lambda_{i}}
$$

where

$$
\lambda_{i}= \begin{cases}u+\mu_{i}, & \text { if } 1 \leq i \leq k-1 ; \\ u, & \text { if } i=k ; \\ u-\mu_{i}, & \text { if } k+1 \leq i \leq n .\end{cases}
$$

It follows that $\lambda=\left(\lambda_{1}, \ldots, \lambda_{n}\right)$ is the index partition of $\psi^{(k)}\left(m^{\prime}\right)$, and the conjugate partition $\lambda^{\prime}$ satisfies

$$
\lambda_{t}^{\prime} \geq k \Longleftrightarrow t \leq u=\mu_{n}
$$

The map $\phi^{(k)}$ replaces, for each $t$ with $\lambda_{t}^{\prime} \geq k$, the product $x_{\pi(1)} \cdots x_{\pi\left(\lambda_{t}^{\prime}\right)}$ by the product $y_{\pi\left(\lambda_{t}^{\prime}+1\right)} \cdots y_{\pi(n)}$. It therefore reduces by $u$ the exponent of each $x_{i}$ with $i \leq k$, removes all the $x_{i}$ with $i \geq k+1$, and replaces them by $y_{i}$ with exponents $u-\lambda_{i}=\mu_{i}$. This implies that

$$
\phi^{(k)}\left(\psi^{(k)}\left(m^{\prime}\right)\right)=\prod_{i=1}^{k-1} x_{\pi(i)}^{\mu_{i}} \cdot \prod_{i=k+1}^{n} y_{\pi(i)}^{\mu_{i}}=m^{\prime},
$$

as claimed. 
Corollary 4.9. The map $\psi^{(k)}: M_{n}^{(k)} \rightarrow M_{n}^{(n)}$ is a bijection.

\subsection{Action and Invariants}

Definition 4.10. For $1 \leq m \leq n-1$ let

$$
e_{m}^{(k)}:= \begin{cases}e_{m}(\bar{x})=e_{m}\left(x_{1}, \ldots, x_{n}\right), & \text { if } 1 \leq m \leq k-1 \\ e_{n-m}(\bar{y})=e_{n-m}\left(y_{1}, \ldots, y_{n}\right), & \text { if } k \leq m \leq n-1,\end{cases}
$$

where $e_{m}(\bar{x})$ is the $m$-th elementary symmetric function.

For a partition $\mu=\left(0<\mu_{1} \leq \cdots \leq \mu_{\ell}\right)$ with $\mu_{\ell}<n$ let

$$
e_{\mu}^{(k)}:=\prod_{i=1}^{\ell} e_{\mu_{i}}^{(k)}
$$

For $e_{\mu}^{(n)}$ we shall frequently use the short notation $e_{\mu}$.

Consider the natural $S_{n}$-action on $\mathcal{P}_{n}^{(k)}$, defined by:

$$
\pi\left(x_{i}\right):=x_{\pi(i)}, \quad \pi\left(y_{i}\right):=y_{\pi(i)}, \quad\left(\forall \pi \in S_{n}, 1 \leq i \leq n\right)
$$

Proposition 4.11. For every $\sigma \in S_{n}$ and $1 \leq k \leq n$,

$$
\sigma \psi^{(k)}=\psi^{(k)} \sigma
$$

Corollary 4.12. $\mathcal{P}_{n}^{(k)}$ and $\mathcal{P}_{n}^{(n)}$ are isomorphic as $S_{n}$-modules.

Let $\mathcal{P}_{n}^{(k)^{S_{n}}}$ be the algebra of $S_{n}$-invariants in $\mathcal{P}_{n}^{(k)}$. Proposition 4.11 implies

Corollary 4.13. For any $1 \leq k \leq n$,

1. $\mathcal{P}_{n}^{(k)^{S_{n}}}$ is generated, as an algebra, by $e_{m}^{(k)}, 1 \leq m<n$.

2. The set $\left\{e_{\mu}^{(k)}: \mu=\left(\mu_{1} \leq \cdots \leq \mu_{\ell}\right)\right.$ with $\left.\mu_{\ell}<n\right\}$ forms a (vector space) basis for $\mathcal{P}_{n}^{(k)^{S_{n}}}$.

3. For every partition $\mu=\left(\mu_{1} \leq \cdots \leq \mu_{\ell}\right)$ with $\mu_{\ell}<n$,

$$
\psi^{(k)}\left(e_{\mu}^{(k)}\right)=e_{\mu} .
$$

Remark 4.14. By Proposition 4.11, $\psi^{(k)}$ and $\phi^{(k)}$ send invariants to invariants. Unfortunately, these maps are not multiplicative and they do not send the ideal generated by invariants (with no constant term) to its analogue. For example, $x_{1} x_{3}+x_{2} x_{3}+x_{3}^{2}=$ $x_{3} \cdot e_{1}\left(x_{1}, x_{2}, x_{3}\right) \in I_{(3)}$ but $\phi^{(2)}\left(x_{1} x_{3}+x_{2} x_{3}+x_{3}^{2}\right)=y_{2}+y_{1}+x_{3}^{2} \notin I_{(2,1)}$. 
In order to define the "correct" map, recall the definitions of the Garsia-Stanton basis element $a_{\pi}$ and the $k$-th descent basis element $a_{\pi}^{(k)}$ corresponding to $\pi \in S_{n}$ (see Definition 1.2).

Claim 4.15. For every $\pi \in S_{n}$ and $1 \leq k \leq n$,

$$
\psi^{(k)}\left(a_{\pi}^{(k)}\right)=a_{\pi} .
$$

In the next section it will be shown that $\left\{a_{\pi}^{(k)} e_{\mu}^{(k)}: \pi \in S_{n}, \mu=\left(\mu_{1} \leq \cdots \leq\right.\right.$ $\left.\mu_{\ell}\right)$ with $\left.\mu_{\ell}<n\right\}$ forms a basis for $\mathcal{P}_{n}^{(k)}$. Thus the map

$$
\tilde{\psi}^{(k)}\left(a_{\pi}^{(k)} \cdot e_{\mu}^{(k)}\right):=\psi^{(k)}\left(a_{\pi}^{(k)}\right) \cdot \psi^{(k)}\left(e_{\mu}^{(k)}\right)=a_{\pi} \cdot e_{\mu}
$$

extends to a linear map $\tilde{\psi}^{(k)}: \mathcal{P}_{n}^{(k)} \rightarrow \mathcal{P}_{n}^{(n)}$ that clearly sends the $k$-th ideal generated by invariants to its $n$-th analogue.

\section{$5 \quad$ Straightening}

\subsection{Basic Notions}

We now generalize several notions and facts which were used in [1] for straightening the coinvariant algebra of type $A$. Some of them were already introduced in the proof of Proposition 4.8 above. Proofs are similar to those in [1], and will be omitted.

Each monomial $m \in M_{n}^{(k)}$ can be written in the form

$$
m=\prod_{i=1}^{k-1} x_{\pi(i)}^{p_{i}} \cdot \prod_{i=k+1}^{n} y_{\pi(i)}^{p_{i}},
$$

where $p_{1} \geq \ldots \geq p_{k-1} \geq 0$ and $0 \leq p_{k+1} \leq \ldots \leq p_{n}$. Here $\pi=\pi(m)$, the index permutation of $m$, is the unique permutation that orders first the indices $i \in \operatorname{Supp}_{x}(m)$, then the indices $i \notin \operatorname{Supp}_{x}(m) \cup \operatorname{Supp}_{y}(m)$, and then the indices $i \in \operatorname{Supp}_{y}(m)$. The $x$ indices are ordered by weakly decreasing exponents, the $y$-indices are ordered by weakly increasing exponents, and indices with equal exponents are ordered in increasing (index) order.

Observation 5.1. The index permutation is preserved by $\psi^{(k)}$, i.e., for any monomial $m \in M_{n}^{(k)}$

$$
\pi(m)=\pi\left(\psi^{(k)}(m)\right) .
$$

Claim 5.2. Let $m$ be a monomial in $M_{n}^{(k)}, \pi=\pi(m)$ its index permutation, and $a_{\pi}^{(k)}$ the corresponding descent basis element:

$$
m=\prod_{i=1}^{k-1} x_{\pi(i)}^{p_{i}} \cdot \prod_{i=k+1}^{n} y_{\pi(i)}^{p_{i}},
$$




$$
a_{\pi}^{(k)}=\prod_{i=1}^{k-1} x_{\pi(i)}^{d_{i}^{(k)}(\pi)} \cdot \prod_{i=k+1}^{n} y_{\pi(i)}^{d_{i}^{(k)}(\pi)}
$$

Then: the sequence $\left(p_{i}-d_{i}^{(k)}(\pi)\right)_{i=1}^{k-1}$ of $x$-exponents in $m / a_{\pi}^{(k)}$ consists of nonnegative integers, and is weakly decreasing:

$$
p_{1}-d_{1}^{(k)}(\pi) \geq \ldots \geq p_{k-1}-d_{k-1}^{(k)}(\pi) \geq 0,
$$

and the sequence $\left(p_{i}-d_{i}^{(k)}(\pi)\right)_{i=k+1}^{n}$ of $y$-exponents $m / a_{\pi}^{(k)}$ consists of nonnegative integers, and is weakly increasing:

$$
0 \leq p_{k+1}-d_{k+1}^{(k)}(\pi) \leq \ldots \leq p_{n}-d_{n}^{(k)}(\pi) .
$$

For a monomial $m \in M_{n}^{(k)}$ of the form (24) with index permutation $\pi \in S_{n}$, let the associated pair of exponent partitions

$$
\lambda(m)=\left(\lambda_{x}(m), \lambda_{y}(m)\right):=\left(\left(p_{1}, p_{2}, \ldots, p_{k-1}\right),\left(p_{n}, p_{n-1}, \ldots, p_{k+1}\right)\right)
$$

be its exponent bipartition. Note that $\lambda(m)$ is a bipartition of the total bi-degree of $m$.

Define the complementary bipartition $\mu^{(k)}(m)=\left(\mu_{x}(m), \mu_{y}(m)\right)$ of $m$ to be the pair of partitions conjugate to the partitions $\left(p_{i}-d_{i}^{(k)}(\pi)\right)_{i=1}^{k-1}$ and $\left(p_{i}-d_{i}^{(k)}(\pi)\right)_{i=n}^{k+1}$ respectively; namely,

$$
\left(\mu_{x}\right)_{j}:=\left|\left\{1 \leq i \leq k-1: p_{i}-d_{i}^{(k)}(\pi) \geq j\right\}\right|
$$

and

$$
\left(\mu_{y}\right)_{j}:=\left|\left\{k+1 \leq i \leq n: p_{i}-d_{i}^{(k)}(\pi) \geq j\right\}\right|
$$

If $k=n$ then, for every monomial $m \in M_{n}^{(n)}, \mu_{y}(m)$ is the empty partition. In this case we denote

$$
\mu(m):=\mu_{x}(m) .
$$

With each $m \in M_{n}^{(k)}$ we associate the canonical complementary partition

$$
\nu(m):=\mu\left(\psi^{(k)}(m)\right)
$$

Example 5.3. Let $m=x_{1}^{2} y_{2}^{4} x_{3}^{2} y_{5} x_{6}^{3}$ with $n=7$ and $k=5$. Then

$$
\begin{gathered}
m=x_{6}^{3} x_{1}^{2} x_{3}^{2} y_{5}^{1} y_{2}^{4}, \quad \lambda(m)=((3,2,2,0),(4,1)), \quad \pi=6134752 \in S_{7}, \\
a_{\pi}^{(5)}=x_{6} y_{5} y_{2}^{2}, \quad \lambda\left(a_{\pi}^{(5)}\right)=((1,0,0,0),(2,1)), \\
\mu^{(5)}(m)=\left((2,2,2,0)^{\prime},(2,0)^{\prime}\right)=((3,3),(1,1)), \\
\psi^{(5)}(m)=x_{6}^{7} x_{1}^{6} x_{3}^{6} x_{4}^{4} x_{7}^{4} x_{5}^{3}, \quad a_{\pi}=x_{6}^{3} x_{1}^{2} x_{3}^{2} x_{4}^{2} x_{7}^{2} x_{5}^{1}, \\
\nu(m)=\mu\left(\psi^{(5)}(m)\right)=(4,4,4,2,2,2)^{\prime}=(6,6,3,3) .
\end{gathered}
$$


Definition 5.4. 1. For two partitions $\lambda$ and $\mu$, denote $\lambda \unlhd \mu$ if $\lambda$ is weakly smaller than $\mu$ in dominance order. For two bipartitions $\lambda^{1}=\left(\mu^{1}, \nu^{1}\right)$ and $\lambda^{2}=\left(\mu^{2}, \nu^{2}\right)$, denote $\lambda^{1} \unlhd \lambda^{2}$ if $\mu^{1} \unlhd \mu^{2}$ and $\nu^{1} \unlhd \nu^{2}$.

2. For two monomials $m_{1}, m_{2} \in M_{n}^{(k)}$ of the same total bi-degree $(p, q)$, write $m_{1} \preceq_{k} m_{2}$ if:

(1) $\lambda\left(m_{1}\right) \unlhd \lambda\left(m_{2}\right)$; and

(2) if $\lambda\left(m_{1}\right)=\lambda\left(m_{2}\right)$ then $\operatorname{inv}\left(\pi\left(m_{1}\right)\right)>\operatorname{inv}\left(\pi\left(m_{2}\right)\right)$.

\subsection{The Straightening Algorithm}

Lemma 5.5. Let $m \in M_{n}^{(k)}$ be a monomial. For $1 \leq d \leq n-1$, let $S_{(d)}$ be the set of all monomials which appear (with coefficient 1) in the expansion of the polynomial $m \cdot e_{d}^{(k)} \in \mathcal{P}_{n}^{(k)}$. Let $\pi=\pi(m)$ be the index permutation of $m$ and denote

$$
m_{(d)}:= \begin{cases}m \cdot x_{\pi(1)} \cdots x_{\pi(d)}, & \text { if } 1 \leq d \leq k-1 \\ m \cdot y_{\pi(d+1)} \cdots y_{\pi(n)}, & \text { if } k \leq d \leq n-1\end{cases}
$$

Then:

(1) $m_{(d)} \in S_{(d)}$.

(2) $\left(m^{\prime} \in S_{(d)}\right.$ and $\left.m^{\prime} \neq m_{(d)}\right) \Longrightarrow m^{\prime} \prec_{k} m_{(d)}$.

The proof of Lemma 5.5 is similar to the proof of [1, Lemma 3.2] and is omitted.

Corollary 5.6. Let $m \in M_{n}^{(k)}$ be a monomial, $\pi=\pi(m)$ its index permutation, and $\nu=\mu\left(\psi^{(k)}(m)\right)$ the canonical complementary partition of $m$ defined in Subsection 5.1. Let $S$ be the set of monomials which appear (with nonzero coefficient) in the expansion of $a_{\pi}^{(k)} \cdot e_{\nu}^{(k)} \in \mathcal{P}_{n}^{(k)}$. Then :

(1) $m \in S$.

(2) $\left(m^{\prime} \in S\right.$ and $\left.m^{\prime} \neq m\right) \Longrightarrow m^{\prime} \prec_{k} m$.

A straightening algorithm follows.

\section{A Straightening Algorithm:}

For a monomial $m \in \mathcal{P}_{n}^{(k)}$, let $\pi=\pi(m)$ be its index permutation, $a_{\pi}^{(k)}$ the corresponding descent basis element, and $\nu=\mu\left(\psi^{(k)}(m)\right)$ the corresponding canonical complementary partition. Write (by Corollary 5.6)

$$
m=a_{\pi}^{(k)} \cdot e_{\nu}^{(k)}-\Sigma,
$$

where $\Sigma$ is a sum of monomials $m^{\prime} \prec_{k} m$. Repeat the process for each $m^{\prime}$.

The algorithm implies 
Lemma 5.7. (Straightening Lemma) Each monomial $m \in \mathcal{P}_{n}^{(k)}$ has an expression

$$
m=a_{\pi(m)}^{(k)} e_{\nu(m)}^{(k)}+\sum_{m^{\prime} \prec_{k} m} n_{m^{\prime}, m} a_{\pi\left(m^{\prime}\right)}^{(k)} e_{\nu\left(m^{\prime}\right)}^{(k)},
$$

where $n_{m^{\prime}, m}$ are integers.

The Straightening Lemma yields a direct proof to the following special case of Theorem 1.5.

Corollary 5.8. The set $\left\{a_{\pi}^{(k)}: \pi \in S_{n}\right\}$ forms a basis for $\mathbf{H}_{\left(1^{k-1}, n-k+1\right)}^{\prime}$.

Proof. Recall that $\mathcal{P}_{n}^{(k)}:=\mathbf{R} / \mathcal{I}_{k}$, where $\mathbf{R}:=\mathbf{Q}\left[x_{1}, \ldots, x_{n}, y_{1}, \ldots, y_{n}\right]$ (Definition 4.1). Let $I_{k}^{0}$ be the ideal of $\mathbf{R}$ generated by the elementary symmetric functions $e_{d}^{(k)}(1 \leq d \leq$ $n-1)$. By definition, these functions are symmetric functions of either $\bar{x}$ or $\bar{y}$. Thus, Corollary 2.3 and Claim 4.2,

$$
\mathcal{I}_{k}+I_{k}^{0} \subseteq J_{\left(1^{k-1}, n-k+1\right)}
$$

By Lemma 5.7, $\left\{a_{\pi}^{(k)}: \pi \in S_{n}\right\}$ spans $\mathbf{R} /\left(\mathcal{I}_{k}+I_{k}^{0}\right)$ and therefore also $\mathbf{R} / J_{\left(1^{k-1}, n-k+1\right)}$ as vector spaces over $\mathbf{Q}$.

On the other hand, by [13], $\operatorname{dim}\left(\mathbf{R} / J_{\left(1^{k-1}, n-k+1\right)}\right)=\left|S_{n}\right|$, and therefore $\left\{a_{\pi}^{(k)}: \pi \in S_{n}\right\}$ is actually a basis for $\mathbf{H}_{\left(1^{k-1}, n-k+1\right)}^{\prime}=\mathbf{R} / J_{\left(1^{k-1}, n-k+1\right)}$.

Second Proof of Theorem 1.4. From the proof of Corollary 5.8 it follows that

$$
\operatorname{dim}\left(\mathbf{R} /\left(\mathcal{I}_{k}+I_{k}^{0}\right)\right)=\operatorname{dim}\left(\mathbf{R} / J_{\left(1^{k-1}, n-k+1\right)}\right) .
$$

Combining this with inclusion (25), it follows that

$$
\mathcal{I}_{k}+I_{k}^{0}=J_{\left(1^{k-1}, n-k+1\right)}
$$

This completes the proof.

\section{Descent Representations}

Recall the definition of the Solomon descent representation $\rho^{A}$ from Subsection 1.4.1. The following theorem is well known. Recall the definition of $\operatorname{Des}(T)$ in Subsection 1.4.2.

Theorem 6.1. For any subset $A \subseteq[n-1]$ and partition $\mu \vdash n$, the multiplicity in the descent representation $\rho^{A}$ of the irreducible $S_{n}$-representation corresponding to $\mu$ is

$$
m_{\mu}^{A}:=\#\{T \in S Y T(\mu): \operatorname{Des}(T)=A\},
$$

the number of standard Young tableaux of shape $\mu$ with descent set $A$. 
Recall the definition of a bipartition and the domination order on bipartitions from Definition 5.4.1. Recall the subspaces $I_{\lambda}^{(k) \unlhd}$ and $I_{\lambda}^{(k) \triangleleft}$ of the $S_{n}$-module $\mathbf{H}_{\left(1^{k-1}, n-k+1\right)}^{\prime}$, and the quotient $R_{\lambda}^{(k)}:=I_{\lambda}^{(k) \unlhd} / I_{\lambda}^{(k) \triangleleft}$, defined in Subsection 1.4.1.

Proposition 6.2. $I_{\lambda}^{(k) \unlhd}, I_{\lambda}^{(k) \triangleleft}$ and thus $R_{\lambda}^{(k)}$ are $S_{n}$-invariant.

Proof. Follows from the straightening algorithm.

Recall that $\lambda=\left(\left(\mu_{1} \geq \cdots \geq \mu_{k}=0\right),\left(\nu_{1} \geq \cdots \geq \nu_{n-k}=0\right)\right)$ is an $(n, k)$ partition, if $\forall(1 \leq i<k-1) \quad\left(\mu_{i}-\mu_{i+1} \in\{0,1\}\right)$ and $\forall(1 \leq i<n-k) \quad\left(\nu_{i}-\nu_{i+1} \in\{0,1\}\right)$.

Lemma 6.3. Let $\lambda=(\mu, \nu)$ be an $(n, k)$-bipartition. Then $R_{\lambda}^{(k)} \neq\{0\}$ and $\left\{a_{\pi}^{(k)}+I_{\lambda}^{(k) \triangleleft}\right.$ : $\left.\operatorname{Des}(\pi)=A_{\lambda}\right\}$ is a basis for $R_{\lambda}^{(k)}$, where $A_{\lambda}$ is defined as in (11).

The proof is analogous to the proof of [1, Corollary 3.10].

Theorem 6.4. The $S_{n}$-action on $R_{\lambda}^{(k)}$ is given by

$$
s_{j}\left(a_{\pi}^{(k)}\right)= \begin{cases}a_{s_{j} \pi}^{(k)}, & \text { if }\left|\pi^{-1}(j+1)-\pi^{-1}(j)\right|>1 ; \\ a_{\pi}^{(k)}, & \text { if } \pi^{-1}(j+1)=\pi^{-1}(j)+1 \\ -a_{\pi}^{(k)}-\sum_{\sigma \in A_{j}(\pi)} a_{\sigma}^{(k)}, & \text { if } \pi^{-1}(j+1)=\pi^{-1}(j)-1 .\end{cases}
$$

Here $s_{j}=(j, j+1)(1 \leq j<n)$ are the Coxeter generators of $S_{n} ;\left\{a_{\pi}^{(k)}+I_{\lambda}^{(k) \triangleleft}: \operatorname{Des}(\pi)=\right.$ $\left.A_{\lambda}\right\}$ is the descent basis of $R_{\lambda}^{(k)}$; for $\pi \in S_{\lambda}$ with $\pi^{-1}(j+1)=\pi^{-1}(j)-1$ we define

$$
\begin{aligned}
t & :=\pi^{-1}(j+1), \\
m_{1} & :=\max \{i \in \operatorname{Des}(\pi) \cup\{0\}: i \leq t-1\}, \\
m_{2} & :=\min \{i \in \operatorname{Des}(\pi) \cup\{n\}: i \geq t+1\}
\end{aligned}
$$

(so that $\pi(t)=j+1, \pi(t+1)=j$, and $\left\{m_{1}+1, \ldots, m_{2}\right\}$ is the maximal interval containing $t$ and $t+1$ on which $s_{j} \pi$ is increasing); and let $A_{j}(\pi)$ be the set of all $\sigma \in S_{n}$ satisfying

1. $\left(i \leq m_{1}\right.$ or $\left.i \geq m_{2}+1\right) \Longrightarrow \sigma(i)=\pi(i)$;

2. the sequences $\left(\sigma\left(m_{1}+1\right), \ldots, \sigma(t)\right)$ and $\left(\sigma(t+1), \ldots, \sigma\left(m_{2}\right)\right)$ are increasing;

3. $\sigma \notin\left\{\pi, s_{j} \pi\right\}$ (i.e., $\{\sigma(t), \sigma(t+1)\} \neq\{j, j+1\}$ ).

Example 6.5. Let $\pi=2416573 \in S_{7}$ and $j=5$. Then:

$$
\begin{gathered}
j=5, j+1=6 ; t=4, t+1=5 ; \\
\operatorname{Des}(\pi)=\{2,4,6\} ; m_{1}=2, m_{2}=6 ; s_{j} \pi=24 \underline{15673} 3 ; \\
A_{j}(\pi)=\{24 \underline{1756} 3,24 \underline{5617} 3,24 \underline{5716} 3,24 \underline{6715} 3\} .
\end{gathered}
$$

Note that $\left|A_{j}(\pi)\right|=\left(\begin{array}{c}m_{2}-m_{1} \\ t-m_{1}\end{array}\right)-2=\left(\begin{array}{l}4 \\ 2\end{array}\right)-2=4$. 
Corollary 6.6. The $S_{n}$-representation on $R_{\lambda}^{(k)}$ is independent of $k$.

Proof of Theorem 6.4. If $\left|\pi^{-1}(j+1)-\pi^{-1}(j)\right|>1$ (i.e., if $j$ and $j+1$ are not adjacent in the sequence $(\pi(1), \ldots, \pi(n)))$, then $\operatorname{Des}\left(s_{j} \pi\right)=\operatorname{Des}(\pi)$ and therefore $s_{j}\left(a_{\pi}^{(k)}\right)=a_{s_{j} \pi}^{(k)}$.

If $\pi^{-1}(j+1)=\pi^{-1}(j)+1$ then $j$ immediately precedes $j+1$ in the sequence $(\pi(1), \ldots, \pi(n))$. Therefore $t:=\pi^{-1}(j) \notin \operatorname{Des}(\pi)$, so that: if $t+1 \leq k-1$ then $x_{j}$ and $x_{j+1}$ have the same exponent in $a_{\pi}^{(k)}$; if $t \geq k+1$ then $y_{j}$ and $y_{j+1}$ have the same exponent in $a_{\pi}^{(k)}$; and if $t \in\{k-1, k\}$ then none of the variables $x_{j}, x_{j+1}, y_{j}$ and $y_{j+1}$ appears in $a_{\pi}^{(k)}$. In all of these cases we have $s_{j}\left(a_{\pi}^{(k)}\right)=a_{\pi}^{(k)}$.

We are left with the most involved case: $\pi^{-1}(j+1)=\pi^{-1}(j)-1$. Recall the notations $t, m_{1}, m_{2}$ and $A_{j}(\pi)$ from the statement of the theorem. In particular, recall that $\pi(t)=$ $j+1, \pi(t+1)=j$, and $\left\{m_{1}+1, \ldots, m_{2}\right\}$ is the maximal interval containing $t$ and $t+1$ on which $s_{j} \pi$ is increasing.

Assume first that $m_{2} \leq k$. The variables $x_{\pi\left(m_{1}+1\right)}, \ldots, x_{\pi\left(m_{2}\right)}$ are all the $x$-variables having the same exponent in $a_{s_{j} \pi}^{(k)}$ as $x_{j}$ (and $x_{j+1}$ ). Since $t \in \operatorname{Des}(\pi)$, the exponents of $x_{\pi(1)}, \ldots, x_{\pi(t)}$ in $a_{\pi}^{(k)}$ are 1 higher than the corresponding exponents in $a_{s_{j} \pi}^{(k)}$. Thus the product $a_{s_{j} \pi}^{(k)} \cdot e_{t}(x)$ (in $\left.\mathcal{P}_{n}^{(k)}\right)$ is a sum of $\left(\begin{array}{c}n \\ t\end{array}\right)$ monomials, one of which is $a_{\pi}^{(k)}$. In all of these monomials, $t$ of the $x$-variables have their exponent increased by 1 (with respect to $\left.a_{s_{j} \pi}^{(k)}\right)$. If these $t$ variables miss any one of $x_{\pi(1)}, \ldots, x_{\pi\left(m_{1}\right)}$, or include any one of $x_{\pi\left(m_{2}+1\right)}, \ldots, x_{\pi(n)}$, then the monomial belongs to $J_{\lambda}^{(k) \triangleleft}$ and contributes nothing to $R_{\lambda}^{(k)}$. The remaining $\left(\begin{array}{c}m_{2}-m_{1} \\ t-m_{1}\end{array}\right)$ monomials are: $a_{\sigma}^{(k)}$ for $\sigma \in A_{j}(\pi), a_{\pi}^{(k)}=a_{s_{j} \pi}^{(k)} \cdot x_{\pi(1)} \cdots x_{\pi(t)}$, and $s_{j}\left(a_{\pi}^{(k)}\right)=a_{s_{j} \pi}^{(k)} \cdot x_{s_{j} \pi(1)} \cdots x_{s_{j} \pi(t)}$. On the other hand, $a_{s_{j} \pi}^{(k)} \cdot e_{t}(x) \equiv 0$ in $R_{\lambda}^{(k)}$ since $1 \leq t \leq m_{2}-1 \leq k-1$. This proves the claim of the theorem in this case.

If $m_{1}+1 \geq k$, then an analogous argument holds for $y$-instead of $x$-variables, and here $a_{s_{j} \pi}^{(k)} \cdot e_{n-t}(y) \equiv 0$ in $R_{\lambda}^{(k)}$ since $1 \leq n-t \leq n-m_{1}-1 \leq n-k$.

Finally, if $m_{1}+1<k<m_{2}$, then the variables $x_{\pi\left(m_{1}+1\right)}, \ldots, x_{\pi\left(m_{2}\right)}$ (and the corresponding $y$-variables) do not appear at all in $a_{s_{j} \pi}^{(k)}$. If $t \leq k-1$ (respectively, $t \geq k$ ) then $a_{s_{j} \pi}^{(k)} \cdot e_{t}(x)$ (respectively, $\left.a_{s_{j} \pi}^{(k)} \cdot e_{n-t}(y)\right)$ is, again, a sum of $\left(\begin{array}{c}m_{2}-m_{1} \\ t-m_{1}\end{array}\right)$ monomials: $a_{\sigma}^{(k)}$ for $\sigma \in A_{j}(\pi), a_{\pi}^{(k)}=a_{s_{j} \pi}^{(k)} \cdot x_{\pi(1)} \cdots x_{\pi(t)}$ (respectively, $\left.a_{\pi}^{(k)}=a_{s_{j} \pi}^{(k)} \cdot y_{\pi(t+1)} \cdots y_{\pi(n)}\right)$, and $s_{j}\left(a_{\pi}^{(k)}\right)=a_{s_{j} \pi}^{(k)} \cdot x_{s_{j} \pi(1)} \cdots x_{s_{j} \pi(t)}$ (respectively, $\left.s_{j}\left(a_{\pi}^{(k)}\right)=a_{s_{j} \pi}^{(k)} \cdot y_{s_{j} \pi(t+1)} \cdots y_{s_{j} \pi(n)}\right)$. This completes the proof.

Theorem 6.7. Let $\lambda=(\mu, \nu)$ be an $(n, k)$-bipartition. $R_{\lambda}^{(k)}$ is isomorphic, as an $S_{n^{-}}$ module, to the Solomon descent representation determined by the descent class $\left\{\pi \in S_{n}\right.$ : $\left.\operatorname{Des}(\pi)=A_{\lambda}\right\}$.

Proof. By Theorem 6.4 together with Lemma 6.3, for every Coxeter generator $s_{i}$, the representation matrices of $s_{i}$ on $R_{\lambda}^{(k)}$ and on $R_{\lambda}^{(n)}$ with respect to the corresponding $k$-th and $n$-th descent monomials respectively are identical. By [1, Theorem 4.1], the multiplicity of the irreducible $S_{n}$-representation corresponding to $\mu$ in $R_{\lambda}^{(n)}$ is $m_{S, \mu}:=\#\{T \in$ 
$\left.S Y T(\mu): \operatorname{Des}(T)=A_{\lambda}\right\}$, the number of standard Young tableaux of shape $\mu$ and descent set $A_{\lambda}$. Theorem 6.1 completes the proof.

Let $R_{t_{1}, t_{2}}^{(k)}$ be the $\left(t_{1}, t_{2}\right)$-th homogeneous component of $\mathbf{H}_{\left(1^{k-1}, n-k+1\right)}^{\prime}$.

Corollary 6.8. For every $t_{1}, t_{2} \geq 0$ and $1 \leq k \leq n$, the $\left(t_{1}, t_{2}\right)$-th homogeneous component of $\mathbf{H}_{\left(1^{k-1}, n-k+1\right)}^{\prime}$ decomposes into a direct sum of Solomon descent representations as follows:

$$
R_{t_{1}, t_{2}}^{(k)} \cong \bigoplus_{\lambda} R_{\lambda}^{(k)}
$$

where the sum is over all $(n, k)$-bipartitions and

$$
\sum_{\nu_{i}>\nu_{i+1} \text { and } i \geq k}(n-i)=t_{1}, \quad \sum_{\mu_{i}>\mu_{i+1} \text { and } i<k} i=t_{2} .
$$

Finally, Theorem 6.7 implies Stembridge's Theorem 1.16.

First Proof of Theorem 1.16. Combine Theorems 6.1 and 6.7 with Corollary 6.8.

\section{The Schur Function Expansion of $\tilde{H}_{\left(1^{k-1}, n-k+1\right)}(\bar{x} ; q, t)$}

In this section we give a direct combinatorial proof of Theorems 1.16 and 1.17, using the axiomatic characterization of Macdonald polynomials and properties of the RSK algorithm.

\subsection{Preliminaries}

A skew Young diagram $\lambda / \mu$ is the difference of Young diagrams $\lambda$ and $\mu \subseteq \lambda$. A skew diagram is a horizontal strip (resp. vertical strip) if it does not contain two cells in the same column (row). A semistandard Young tableau of (skew) shape $\lambda$ is a function $T$ from the diagram of $\lambda$ to the ordered alphabet

$$
A_{+}=\{1<2<\ldots\}
$$

which is weakly increasing in each row of $\lambda$ from left to right and strictly increasing in each column from bottom to top. A semistandard tableau is standard if it is a bijection form $\lambda$ to $\{1, \ldots, n=|\lambda|\}$. More generally, we shall admit the ordered alphabet

$$
A_{ \pm}=A_{+} \cup A_{-}=\{1<\overline{1}<2<\overline{2}<\cdots\}
$$

of positive letters $1,2, \ldots$ and negative letters $\overline{1}, \overline{2}, \ldots$ For any alphabet $B$, we let $B^{*}$ denote the set of all words over $B$ and $B^{n}$ denote the set of all words $w \in B^{*}$ of length $n$.

A super tableau is a function $T: \lambda \rightarrow A_{ \pm}$which is weakly increasing in each row and column and is such that the entries equal to $a$ occupy a horizontal strip if $a$ is positive 
and a vertical strip if $a$ is negative. Thus a semistandard tableaux is just a super tableau where all the entries are positive. We denote

$S S Y T(\lambda)=\left\{\right.$ semistandard tableaux $\left.T: \lambda \rightarrow A_{+}\right\}$

$S S Y T_{ \pm}(\lambda)=\left\{\right.$ super tableaux $\left.T: \lambda \rightarrow A_{ \pm}\right\}$

$\operatorname{SSYT}(\lambda, \mu)=\left\{\right.$ semistandard tableaux $T: \lambda \rightarrow A_{+}$with entries $\left.1^{\mu_{1}}, 2^{\mu_{2}}, \ldots\right\}$,

$S S Y T_{ \pm}(\lambda, \mu, \nu)=\left\{\right.$ semistandard super tableaux $T: \lambda \rightarrow A_{+}$with entries $\left.1^{\mu_{1}}, \overline{1}^{\nu_{1}}, \ldots\right\}$,

$S Y T(\lambda)=S S Y T\left(\lambda, 1^{n}\right)=\{$ standard tableaux $T: \lambda \rightarrow\{1, \ldots, n=|\lambda|\}\}$.

If $T$ is any one of these types of tableaux, we shall let $\operatorname{sh}(T)=\lambda$ denote the shape of $T, \operatorname{pos}(T)$ denote the number of positive letters in the range of $T$ and neg $(T)$ denote the number of negative letters in the range of $T$.

We write $\langle$,$\rangle for the Hall inner product on symmetric functions, defined by either$ one of the identities

$$
\left\langle h_{\lambda}, m_{\mu}\right\rangle=\delta_{\lambda, \mu}=\left\langle s_{\lambda}, s_{\mu}\right\rangle .
$$

We denote by $\omega$ the involution defined by either one of the identities

$$
\omega\left(h_{\lambda}\right)=e_{\lambda}, \omega\left(e_{\lambda}\right)=h_{\lambda}, \omega\left(s_{\lambda}\right)=s_{\lambda^{\prime}}
$$

We shall use square brackets $f[A]$ to denote the plethystic evaluation of a symmetric function $f$ at a polynomial, rational function, or formal power series $A$. This is defined by writing $f$ in terms of the power symmetric functions and then substituting $p_{m}[A]$ for $p_{m}(\bar{x})$, where $p_{m}[A]$ is the result of substituting $a \rightarrow a^{m}$ for every indeterminate $a$. The standard $\lambda$-ring identities hold for plethystic evaluation, e.g. $s_{\lambda}[X+Y]=\sum_{\mu \subseteq \lambda} s_{\mu}[X] s_{\lambda / \mu}[Y]$, etc. In particular, setting $Z=z_{1}+z_{2}+\cdots$, we have $f[Z]=f\left(z_{1}, z_{2}, \ldots\right)$.

If $W=w_{1}+w_{2}+\ldots$ and $f$ is a symmetric function, we shall use the notation

$$
\omega^{W} f[Z+W]
$$

to denote the result of applying $\omega$ to $f[Z+W]=f\left(z_{1}, z_{2}, \ldots, w_{1}, w_{2} \ldots\right)$ considered as a symmetric function in the $w$ variables with functions of $z$ as coefficients. Equations (27) and (28) then imply that the coefficient of a monomial $z^{\mu} w^{\eta}=z_{1}^{\mu_{1}} z_{2}^{\mu_{2}} \cdots w_{1}^{\eta_{1}} w_{2}^{\eta_{2}} \cdots$ in $\omega^{W} f[Z+W]$ is given by

$$
\left.\omega^{W} f[Z+W]\right|_{z^{\mu} w^{\eta}}=\left\langle f, e_{\eta}(z) h_{\mu}(w)\right\rangle .
$$

If $T$ is a semistandard tableau of (skew) shape $\lambda$, we set

$$
z^{T}=\prod_{x \in \lambda} z_{T(x)}
$$

Then the usual combinatorial definition of the Schur function $s_{\lambda}$ is

$$
s_{\lambda}\left(z_{1}, z_{2}, \ldots\right)=\sum_{T \in S S Y T(\lambda)} z^{T}
$$


Throughout what follows, we fix

$$
Z=z_{1}+z_{2}+\cdots, W=w_{1}+w_{2}+\cdots,
$$

and make the convention that

$$
z_{\bar{a}} \text { stands for } w_{a} \text {, for every negative letter } \bar{a} \in A_{-} \text {. }
$$

Then the "super" analogue of (31) is

$$
H S_{\lambda}(z, w)=\omega^{W} s_{\lambda}[Z+W]=\sum_{T \in S S Y T_{ \pm}(\lambda)} z^{T} .
$$

We shall refer to $H S_{\lambda}(z, w)$ as the super Schur function, but it is also called a hook Schur function $H S_{\lambda}(z, w)$ by Berele and Regev [8]. We note that the right hand side of (32) is actually independent of the relative order of positive letters $A_{+}$and negative letters $A_{-}$. That is, if $A_{+}$is ordered $1<2<\cdots$ and $A_{-}$is ordered $\overline{1}<\overline{2}<$, then the RHS of (32) is independent of the relative order between the positive and negative letters. Thus, for example, if we let $1<2<\cdots<\overline{1}<\overline{2}<\cdots$, the super tableaux $T$ of shape $\lambda$ will consists of a pair of tableau $\left(T_{1}, T_{2}\right)$ where $T_{1}: \mu \rightarrow A_{+}$is a semistandard tableau of shape $\mu$ for some $\mu \subseteq \lambda$ and $T_{2}: \lambda^{\prime} / \mu^{\prime} \rightarrow A_{-}$is a semi-standard tableau of shape $\lambda^{\prime} / \mu^{\prime}$ or, equivalently, we can think of $T_{2}$ as a row strict tableau of shape $\lambda / \mu$ by conjugation. Thus we can think of $T$ as a filling of $\lambda$ with positive and negative numbers such that positive numbers form a column strict tableau of shape $\mu$ and the negative numbers form a row strict tableau of shape $\lambda / \mu$. Given such a $T$, we can achieve a super tableau $T^{\prime}$ from $T$ corresponding to any other relative order between $A_{+}$and $A_{-}$by using jeu de taquin to move the negative numbers past the positive numbers as was done in $[30,31]$. Another proof of the independence of the RHS of (31) of the relative order of the positive and negative letters can be found in [29]. It follows that

$$
H S_{\lambda}(z, w)=\sum_{\mu \subseteq \lambda} s_{\mu}[Z] s_{\lambda^{\prime} / \mu^{\prime}}[W]
$$

Similarly, it follows that the RHS of (32) is also independent of the relative order of the positive letters among themselves and the relative order of the negative letters among themselves.

\subsection{Second Proof of Theorem 1.16}

As noted in $[21]$, the set $\left\{\tilde{H}_{\mu}[Z ; q, t]\right\}_{\mu \vdash n}$ can be characterized as the unique basis of the space of homogeneous symmetric polynomials of degree $n$ over the field $F=\mathbf{Q}(q, t)$ of rational functions in $q$ and $t$, that satisfies the following three properties:

A1 $\tilde{H}_{\mu}[Z(q-1) ; q, t]=\sum_{\rho \unlhd \mu^{\prime}} c_{\rho, \mu} m_{\rho}[Z]$.

A2 $\tilde{H}_{\mu}[Z(t-1) ; q, t]=\sum_{\rho \unlhd \mu} d_{\rho, \mu} m_{\rho}[Z]$. 
A3 $\left.\tilde{H}_{\mu}\left[z_{1}, \ldots, z_{n} ; q, t\right]\right|_{z_{1}^{n}}=1$.

where $\unlhd$ denotes the dominance order.

The row insertion algorithm of the Robinson-Schensted-Knuth (RSK) correspondence has the property that if $a_{1} \cdots a_{n}$ is a sequence of positive letters and

$$
a_{1} \cdots a_{n} \rightarrow_{R S K}(P, Q)
$$

where $P$ is a semistandard Young tableau, $Q$ is a standard tableau, $\operatorname{sh}(P)=\operatorname{sh}(Q)$, and

1. if $a_{i} \leq a_{i+1}$, then $i+1$ lies strictly to right and weakly below $i$ in $Q$ and

2. if $a_{i}>a_{i+1}$, then $i+1$ lies strictly above and weakly to the left of $i$ in $Q$.

Given a sequence of positive letters $a=a_{1} \ldots a_{n}$, some $1 \leq k \leq n$, and some linear order $\prec$ on $A_{+}$, we let (in analogy with the corresponding definitions for tableaux in Subsection 1.4.2 above)

$$
\operatorname{maj}_{1, k}(a, \prec)=\sum_{1 \leq i<k, a_{i} \succ a_{i+1}} i
$$

and

$$
\operatorname{comaj}_{k, n}(a, \prec)=\sum_{k \leq i<n, a_{i} \succ a_{i+1}}(n-i) .
$$

It then follows from Theorem 1.16 and the properties of the RSK algorithm that

$$
\begin{aligned}
\tilde{H}_{\left(1^{k-1}, n-k+1\right)}[Z ; q, t] & =\sum_{\lambda \vdash n} s_{\lambda}[Z] \tilde{K}_{\lambda,\left(1^{k-1}, n-k+1\right)}(q, t) \\
& =\sum_{\lambda \vdash n} \sum_{P \in S S Y T(\lambda)} z^{P} \sum_{Q \in S Y T(\lambda)} q^{\operatorname{maj}_{1, n-k+1}(Q)} t^{\mathrm{comaj}_{n-k+1, n}(Q)} \\
& =\sum_{a=a_{1} \cdots a_{n} \in A_{ \pm}^{n}} z_{a_{1}} \cdots z_{a_{n}} q^{\operatorname{maj}_{1, n-k+1}(a, \prec)} t^{\operatorname{comaj}_{n-k+1, n}(a, \prec)} .
\end{aligned}
$$

To prove (A1) and (A2), we need to interpret $\tilde{H}_{\mu}[Z(q-1)]$ and $\tilde{H}_{\mu}[Z(t-1)]$. Note that

$$
\begin{aligned}
\tilde{H}_{\mu}[Z(q-1)] & =\sum_{\lambda \vdash|\mu|} s_{\lambda}[q Z-Z] \tilde{K}_{\lambda, \mu}(q, t) \\
& =\sum_{\lambda \vdash|\mu|} \tilde{K}_{\lambda, \mu}(q, t) \sum_{\nu \subseteq \lambda} s_{\nu}[q Z](-1)^{|\lambda / \nu|} s_{\lambda^{\prime} / \nu^{\prime}}[Z] .
\end{aligned}
$$

Here we have used the $\lambda$-ring identity

$$
s_{\lambda}[X-Y]=\sum_{\nu \subseteq \lambda} s_{\nu}[X](-1)^{|\lambda / \nu|} s_{\lambda^{\prime} / \nu^{\prime}}[Y] .
$$


Our goal is to get an interpretation of $\tilde{H}_{\left(1^{k}, n-k\right)}[Z(1-q)]$ in terms of statistics on words which is similar to (36). To this end, we shall consider an extension of the RSK algorithm to words over $A_{ \pm}$where we row insert positive letters and dual row insert negative letters as in [30]. Recall that in the dual row insertion algorithm of the Robinson-SchenstedKnuth (DRSK) correspondence, an $x$ bumps the first element which is greater than or equal to $x$ in a row into which $x$ is inserted as opposed to $x$ bumping the first element which is greater than $x$ in a row into which $x$ inserted in row insertion algorithm. The DRSK algorithm has the property that that if $\bar{a}_{1} \cdots \bar{a}_{n}$ is a sequence of negative letters and

$$
\bar{a}_{1} \cdots \bar{a}_{n} \rightarrow_{D R S K}(P, Q)
$$

where the transpose of $P, P^{T}$, is a semistandard Young tableau, $Q$ is a standard tableau, and $\operatorname{sh}(P)=\operatorname{sh}(Q)$, then

1. if $\bar{a}_{i}<\bar{a}_{i+1}$, then $i+1$ lies strictly to right and weakly below $i$ in $Q$ and

2. if $\bar{a}_{i} \geq \bar{a}_{i+1}$, then $i+1$ lies strictly above and weakly to the left of $i$ in $Q$.

Given a word $w=w_{1} \cdots w_{n} \in A_{ \pm}^{n}, 1 \leq k \leq n$, and some fixed linear order $\prec$ on $A_{ \pm}$, we shall let

1. neg $(w)$ be the number of negative letters in $w$,

2. $\operatorname{pos}(w)$ be the number of positive letters in $w$,

3. $\operatorname{Des}(w, \prec)$ be the set of all $i$ such that

(i) $a_{i} \succeq a_{i+1}$ if both $a_{i}$ and $a_{i+1}$ are negative and

(ii) $a_{i} \succ a_{i+1}$ otherwise;

4. $\operatorname{maj}_{1, k}(w, \prec)=\sum_{1 \leq i<k, i \in \operatorname{Des}(a, \prec)} i$, and

5. $\operatorname{comaj}_{k, n}=\sum_{k \leq i<n, i \in \operatorname{Des}(a, \prec)}(n-i)$.

This given, to prove Stembridge's Theorem 1.16 directly, we shall consider the following family of polynomials $\left\{\bar{H}_{\mu}[Z ; q, t]\right\}_{\mu \vdash n}$ where

(a) $\bar{H}_{\mu}[Z ; q, t]=\tilde{H}_{\mu}[Z ; q, t]$ if $\mu \neq\left(1^{k-1}, n-k+1\right)$ and

(b)

$$
\begin{aligned}
\bar{H}_{\left(1^{k-1}, n-k+1\right)}[Z ; q, t] & =\sum_{a=a_{1} \cdots a_{n} \in A_{+}^{n}} z_{a_{1}} \cdots z_{a_{n}} q^{\mathrm{maj}_{1, n-k+1}(a, \prec)} t^{\mathrm{comaj}_{n-k+1, n}(a, \prec)} \\
& =\sum_{\lambda \vdash n} \sum_{P \in S S Y T(\lambda)} \sum_{Q \in S Y T(\lambda)} z^{P} q^{\mathrm{maj}_{1, n-k+1}(Q)} t^{\mathrm{comaj}_{n-k+1, n}(Q)} \\
& =\sum_{\lambda \vdash n} s_{\lambda}[Z] \sum_{Q \in S Y T(\lambda)} q^{\mathrm{maj}_{1, n-k+1}(Q)} t^{\mathrm{comaj}_{n-k+1, n}(Q)}
\end{aligned}
$$


To prove that $\bar{H}_{\mu}[Z ; q, t]=\tilde{H}_{\mu}[Z ; q, t]$ for all $\mu$, we need only prove that the family of polynomials $\left\{\bar{H}_{\mu}[Z ; q, t]\right\}_{\mu \vdash n}$ satisfies the analogues of (A1), (A2), and (A3). Clearly from our definitions, we need only show that $\bar{H}_{\left(1^{k-1}, n-k+1\right)}[Z ; q, t]$ satisfies the analogues of (A1), (A2), and (A3).

It immediately follows from (38) that the analogue of (A3) holds since the only word contributing to the coefficient of $z_{1}^{n}$ is the word $a=1^{n}$ and clearly $\operatorname{maj}_{1, n-k+1}(a, \prec)=$ comaj $_{n-k+1, n}(a, \prec)=0$ for all $\prec$ in this case. Thus $\left.\bar{H}_{\left(1^{k-1}, n-k+1\right)}[Z ; q, t]\right|_{z_{1}^{n}}=1$ as desired.

It follows from the properties of the row insertion and dual row insertion of the RSK correspondence described above that

$$
\begin{aligned}
& \bar{H}_{\left(1^{k}, n-k\right)}[Z(q-1) ; q, t] \\
& =\sum_{\lambda \vdash n}\left(\sum_{Q \in S Y T(\lambda)} q^{\mathrm{maj}_{1, n-k+1}(Q)} t^{\mathrm{comaj}_{n-k+1, n}(Q)}\right) \sum_{\nu \subseteq \lambda} q^{|\nu|} s_{\nu}[Z](-1)^{|\lambda / \nu|} s_{\lambda^{\prime} / \nu^{\prime}}[Z] \\
& =\sum_{\lambda \vdash n} \sum_{P \in S S Y T_{ \pm}(\lambda)} \sum_{Q \in S Y T(\lambda)}(-1)^{n e g(P)} q^{p o s(P)} z^{P} q^{\text {maj }_{1, n-k+1}(Q)} t^{\mathrm{comaj}_{n-k+1, n}(Q)} \\
& =\sum_{a=a_{1} \cdots a_{n} \in A_{ \pm}^{n}} q^{p o s(a)}(-1)^{n e g(a)} z_{\left|a_{1}\right|} \cdots z_{\left|a_{n}\right|} q^{\mathrm{maj}_{1, n-k+1}(a, \prec)} t^{\mathrm{comaj}_{n-k+1, n}(a, \prec)}
\end{aligned}
$$

for any linear order $\prec$ on $A_{ \pm}$. Similarly,

$$
\begin{aligned}
& \bar{H}_{\left(1^{k}, n-k\right)}[Z(t-1) ; q, t] \\
& =\sum_{\lambda \vdash n}\left(\sum_{Q \in S Y T(\lambda)} q^{\operatorname{maj}_{1, n-k+1}(Q)} t^{\mathrm{comaj}_{n-k+1, n}(Q)}\right) s_{\lambda}[t Z-Z] \\
& =\sum_{\lambda \vdash n}\left(\sum_{Q \in S Y T(\lambda)} q^{\operatorname{maj}_{1, n-k+1}(Q)} t^{\mathrm{comaj}_{n-k+1, n}(Q)}\right) \sum_{\nu \subseteq \lambda} t^{|\nu|} s_{\nu}[Z](-1)^{|\lambda / \nu|} s_{\lambda^{\prime} / \nu^{\prime}}[Z] \\
& =\sum_{\lambda \vdash n} \sum_{P \in S S Y T_{ \pm}(\lambda)} \sum_{Q \in S Y T(\lambda)}(-1)^{n e g(P)} t^{\text {pos }(P)} z^{P} q^{\text {maj }_{1, n-k+1}(Q)} t^{\operatorname{comaj}_{n-k+1, n}(Q)} \\
& =\sum_{a=a_{1} \cdots a_{n} \in A_{ \pm}^{n}} t^{\operatorname{pos}^{n}(a)}(-1)^{n e g(a)} z_{\left|a_{1}\right|} \cdots z_{\left|a_{n}\right|} q^{\operatorname{maj}_{1, n-k+1}(a, \prec)} t^{\operatorname{comaj}_{n-k+1, n}(a, \prec)}
\end{aligned}
$$

for any linear order $\prec$ on $A_{ \pm}$.

Thus to prove (A1), we must prove that there exists a linear order $\prec$ on $A_{ \pm}$such that

$$
\begin{aligned}
& \sum_{a=a_{1} \cdots a_{n} \in A_{ \pm}^{n}} q^{\text {pos(a)}}(-1)^{n e g(a)} z_{\left|a_{1}\right|} \cdots z_{\left|a_{n}\right|} q^{\operatorname{maj}_{1, n-k+1}(a, \prec)} t^{\operatorname{comaj}_{n-k+1, n}(a, \prec)} \\
& \quad=\sum_{\rho \unlhd\left(1^{n-k}, k\right)} c_{\rho,\left(1^{k-1}, n-k+1\right)} m_{\rho} .
\end{aligned}
$$


Similarly to prove (A2), we must prove that there exists a linear order $\prec$ on $A_{ \pm}$such that

$$
\begin{aligned}
& \sum_{a=a_{1} \cdots a_{n} \in A_{ \pm}^{n}} t^{\operatorname{pos}(a)}(-1)^{\operatorname{neg}(a)} z_{\left|a_{1}\right|} \cdots z_{\left|a_{n}\right|} q^{\operatorname{maj}_{1, n-k+1}(a, \prec)} t^{\operatorname{comaj}_{n-k+1, n}(a, \prec)} \\
& \quad=\sum_{\rho \unlhd\left(1^{k-1}, n-k+1\right)} d_{\rho,\left(1^{k-1}, n-k+1\right)} m_{\rho}
\end{aligned}
$$

for some $c_{\rho,\left(1^{k-1}, n-k+1\right)}, d_{\rho,\left(1^{k-1}, n-k+1\right)} \in Q(q, t)$.

We shall prove (42) and (43) via simple involutions. We shall start with proving (42). For any ordering $\prec$, define the weight $U(a)$ of a word $a=a_{1} \cdots a_{n} \in A_{ \pm}^{n}$ by setting

$$
U(a)=q^{\operatorname{pos}(a)}(-1)^{\text {neg(a) }} z_{\left|a_{1}\right|} \cdots z_{\left|a_{n}\right|} q^{\operatorname{maj}_{1, n-k+1}(a, \prec)} t^{\operatorname{comaj}_{n-k+1, n}(a, \prec)} .
$$

First we define $\prec$ so that

$$
1 \prec 2 \prec \cdots \prec n \prec \bar{n} \prec \overline{n-1} \prec \cdots \prec \overline{1} .
$$

Then we define an involution $I_{k}$ on $A_{ \pm}^{n}$ as follows. We let $|i|=|\bar{i}|=i$. Given a word $w=a_{1} \cdots a_{n} \in A_{ \pm}^{n}$, we look for the smallest letter $j$ which is repeated in $\left|a_{1}\right| \cdots\left|a_{n-k+1}\right|$. If there is no such letter, we let $I_{k}(w)=w$. Otherwise, let $a_{t}$ be right most occurrence of either $j$ or $\bar{j}$ in $a_{1} \cdots a_{n-k+1}$. Thus $1<t \leq n-k+1$. Now let $i$ be the smallest letter in $\left|a_{1}\right| \cdots\left|a_{t-1}\right|$ and let $a_{s}$ be the left most occurrence of $i$ or $\bar{i}$ in $a_{1} \ldots a_{t-1}$. Note that $1 \leq s \leq n-k$ since $t \leq n-k+1$. Then $I_{k}(a)=b=b_{1} \cdots b_{n}$ where (i) $b_{r}=a_{r}$ if $r \neq s$, (ii) $b_{s}=i$ if $a_{s}=\bar{i}$, and (iii) $b_{s}=\bar{i}$ if $a_{s}=i$. In other words, $I_{k}(a)$ is the result of changing $a_{s}$ to $\bar{i}$ if $a_{s}=i$ or changing $a_{s}$ to $i$ if $a_{s}=\bar{i}$. Clearly $I_{k}^{2}$ is the identity. We claim that $U(a)=-U(b)$. Clearly $(-1)^{\text {neg(a) }}=-(-1)^{\text {neg(b) }}$ since we changed the sign of one letter. We did not change the absolute value of any letter so that $z_{\left|a_{1}\right|} \cdots z_{\left|a_{n}\right|}=z_{\left|b_{1}\right|} \cdots z_{\left|b_{n}\right|}$. Since we did not change any of the letters after place $n-k$, we have $t^{\text {comaj }_{n-k+1, n}(a, \prec)}=t^{\operatorname{comaj}_{n-k+1, n}(b, \prec)}$. Thus we need only show that

$$
q^{\text {pos(a) }} q^{\operatorname{maj}_{1, n-k+1}(a, \prec)}=q^{\text {pos(b) }} q^{\operatorname{maj}_{1, n-k+1}(b, \prec)}
$$

Without loss of generality, we may assume that $a_{s}=i$. Then there are two cases.

Case 1. $s=1$. Since $i$ is the smallest element in $\left|a_{1}\right| \cdots\left|a_{t}\right|$, it follows $i \preceq a_{2}$ and hence $1 \notin \operatorname{Des}(a, \prec)$. We claim that $1 \in \operatorname{Des}(b, \prec)$. Now if $\left|a_{2}\right|>i$ or $a_{2}=i$, then clearly $\bar{i} \succ a_{2}$ by our choice of the order $\prec$ so that $1 \in \operatorname{Des}(b, \prec)$. Finally if $a_{2}=\bar{i}$, then $1 \in \operatorname{Des}(b, \prec)$ by our definition of $\operatorname{Des}(a, \prec)$ since we have two consecutive equal negative numbers. Thus it follows that $\operatorname{maj}_{1, n-k+1}(b, \prec)-1=\operatorname{maj}_{1, n-k+1}(a, \prec)$ and hence

$$
q^{\text {pos }(a)} q^{\text {maj }_{1, n-k+1}(a, \prec)}=q^{\text {pos(b)+1 }} q^{\operatorname{maj}_{1, n-k+1}(b, \prec)-1}=q^{\text {pos }(b)} q^{\operatorname{maj}_{1, n-k+1}(b, \prec)}
$$

as desired.

Case 2. $s>1$. We can argue exactly as in Case 1 that $s \notin D e s(a, \prec)$ and $s \in D e s(b, \prec)$. 
So consider $a_{s-1}$. Our choice of $i$ and $s$ ensures that $\left|a_{s-1}\right|$ is strictly greater than $i$. Thus by our definition of $\prec, i \prec a_{s-1} \prec \bar{i}$. Hence $s-1 \in \operatorname{Des}(a, \prec)$ and $s-1 \notin \operatorname{Des}(b, \prec)$. Thus it again follows that $\operatorname{maj}_{1, n-k+1}(b, \prec)-1=\operatorname{maj}_{1, n-k+1}(a, \prec)$ and hence

$$
q^{\text {pos }(a)} q^{\mathrm{maj}_{1, n-k+1}(a, \prec)}=q^{\text {pos(b)+1}} q^{\mathrm{maj}_{1, n-k+1}(b, \prec)-1}=q^{\text {pos(b) }} q^{\operatorname{maj}_{1, n-k+1}(b, \prec)}
$$

as desired.

Thus our involution $I_{k}$ shows that

$$
\begin{aligned}
& \sum_{a=a_{1} \cdots a_{n} \in A_{ \pm}^{n}} q^{\text {pos(a) }}(-1)^{n e g(a)} z_{\left|a_{1}\right|} \cdots z_{\left|a_{n}\right|} q^{\text {maj }_{1, n-k+1}(a, \prec)} t^{\operatorname{comaj}_{n-k+1, n}(a, \prec)} \\
= & \sum_{a=a_{1} \cdots a_{n} \in A_{ \pm}^{n}, I_{k}(a)=a} q^{\text {pos(a)}}(-1)^{n e g(a)} z_{\left|a_{1}\right|} \cdots z_{\left|a_{n}\right|} q^{\text {maj }_{1, n-k+1}(a, \prec)} t^{\operatorname{comaj}_{n-k+1, n}(a, \prec)} .
\end{aligned}
$$

But since the only words $a=a_{1} \cdots a_{n}$ such that $I_{k}(a)=a$ are those where $\left|a_{1}\right| \cdots\left|a_{n-k+1}\right|$ are pairwise distinct, it follows that the largest possible type of a monomial $z_{\left|a_{1}\right|} \cdots z_{\left|a_{n}\right|}$ relative to the dominance order is $\left(1^{n-k}, k\right)$ since this is largest type of words with at least $k+1$ distinct letters. Thus (42) holds.

The proof of (43) is similar to the proof of (42). First we define $\prec^{*}$ so that

$$
\overline{1} \prec^{*} \overline{2} \prec^{*} \ldots \prec^{*} \bar{n} \prec^{*} n \prec^{*} n-1 \prec^{*} \ldots \prec^{*} 1 .
$$

Then we define the weight $V(a)$ of a word $a=a_{1} \cdots a_{n} \in A_{ \pm}^{n}$ by setting

$$
V(a)=t^{\operatorname{pos}(a)}(-1)^{n e g(a)} z_{\left|a_{1}\right|} \cdots z_{\left|a_{n}\right|} q^{\operatorname{maj}_{1, n-k+1}\left(a, \prec^{*}\right)} t^{\operatorname{comaj}_{n-k+1, n}\left(a, \prec^{*}\right)} .
$$

We define an involution $J_{k}$ on $A_{ \pm}^{n}$ as follows. Given a word $a=a_{1} \cdots a_{n} \in A_{ \pm}^{n}$, we look for the smallest letter $j$ which is repeated in $\left|a_{n-k+1}\right| \cdots\left|a_{n}\right|$. If there is no such letter, we let $J_{k}(a)=a$. Otherwise, let $a_{t}$ be the left most occurrence of either $j$ or $\bar{j}$ in $a_{n-k+1} \cdots a_{n}$. Now let $i$ be the smallest letter in $\left|a_{t+1}\right| \cdots\left|a_{n}\right|$ and let $s$ be the right most occurrence of either $i$ or $\bar{i}$ in $a_{t+1} \ldots a_{n}$.

Thus $n-k+1<t+1 \leq s$ since $n-k+1 \leq t$. Then $J_{k}(a)=b=b_{1} \cdots b_{n}$ where (i) $b_{r}=a_{r}$ if $r \neq s$, (ii) $b_{s}=i$ if $a_{s}=\bar{i}$, and (iii) $b_{s}=\bar{i}$ if $a_{s}=i$. In other words, $J_{k}(a)$ is the result of changing $a_{s}$ to $\bar{i}$ if $a_{s}=i$ or changing $a_{s}$ to $i$ if $a_{s}=\bar{i}$. Clearly $J_{k}^{2}$ is the identity. We claim that $V(a)=-V(b)$. Clearly $(-1)^{\text {neg(a) }}=-(-1)^{\text {neg(b) }}$ since we changed the sign of one letter. Again we did not change the absolute value of any letter so that $z_{\left|a_{1}\right|} \cdots z_{\left|a_{n}\right|}=z_{\left|b_{1}\right|} \cdots z_{\left|b_{n}\right|}$. We did not change any of the letters among $a_{1} \cdots a_{n-k+1}$ so that $q^{\text {maj }_{1, n-k+1}\left(a, \prec^{*}\right)}=q^{\text {maj }_{1, n-k+1}\left(b, \prec^{*}\right)}$. Thus we need only show that

$$
t^{p o s(a)} t^{\mathrm{comaj}_{n-k+1, n}\left(a, \prec^{*}\right)}=t^{\text {pos(b) }} t^{\mathrm{comaj}_{n-k+1, n}\left(b, \prec^{*}\right)}
$$

There is no loss of generality in assuming that $a_{s}=i$. Then there are two cases.

Case 1. $s=n$. Since $i$ is the smallest element in $\left|a_{t}\right| \cdots\left|a_{n}\right|$, it follows $a_{n-1} \preceq^{*} i$. We claim that $n-1 \notin \operatorname{Des}\left(a, \prec^{*}\right)$ and that $n-1 \in \operatorname{Des}\left(b, \prec^{*}\right)$. Now if $\left|a_{n-1}\right|>i$, then clearly 
$\bar{i} \prec^{*} a_{n-1} \prec^{*} i$ so that our claim holds. If $a_{n-1}=i$, then $n-1 \notin \operatorname{Des}\left(a, \prec^{*}\right)$ because two equal positive letters do not cause a descent by our definitions. However, $\bar{i} \prec^{*} i$ so that $n-1 \in \operatorname{Des}\left(b, \prec^{*}\right)$. Finally if $a_{n-1}=\bar{i}$, then $n-1 \in \operatorname{Des}\left(b, \prec^{*}\right)$ since two equal negative letters cause a descent while $n-1 \notin \operatorname{Des}\left(a, \prec^{*}\right)$ because $\bar{i} \prec^{*} i$. Thus it follows that $\operatorname{comaj}_{n-k+1, n}\left(b, \prec^{*}\right)-1=\operatorname{comaj}_{n-k+1, n}\left(a, \prec^{*}\right)$ and hence

$$
t^{p o s(a)} c^{\operatorname{comaj}_{n-k+1, n}\left(a, \prec^{*}\right)}=t^{p o s(b)+1} t^{\operatorname{comaj}_{n-k+1, n}\left(b, \prec^{*}\right)-1}=t^{p o s(b)} t^{\operatorname{comaj}_{n-k+1, n}\left(b, \prec^{*}\right)}
$$

as desired.

Case 2. $s<n$. We can argue exactly as in Case 1 that $s-1 \notin \operatorname{Des}\left(a, \prec^{*}\right)$ and $s-1 \in \operatorname{Des}\left(b, \prec^{*}\right)$. So consider $a_{s+1}$. Our choice of $i$ and $s$ ensures that $\left|a_{s+1}\right|$ is strictly greater than $i$. Thus by our definition of $\prec^{*}, \bar{i} \prec^{*} a_{s+1} \prec^{*} i$. Hence $s \in \operatorname{Des}\left(a, \prec^{*}\right)$ and $s \notin \operatorname{Des}\left(b, \prec^{*}\right)$. Thus the only places were $\operatorname{Des}\left(a, \prec^{*}\right)$ and $\operatorname{Des}\left(a, \prec^{*}\right)$ differ is on the set $\{s-1, s\}$. We have $\operatorname{Des}\left(a, \prec^{*}\right) \cap\{s-1, s\}=\{s\}$ and $\operatorname{Des}\left(b, \prec^{*}\right) \cap\{s-1, s\}=\{s-1\}$. Since $n-(s-1)=(n-s)+1$, it follows that $\operatorname{comaj}_{n-k+1, n}\left(b, \prec^{*}\right)-1=\operatorname{comaj}_{n-k+1, n}\left(a, \prec^{*}\right)$ and hence

$$
t^{p o s(a)} t^{\operatorname{comaj}_{n-k+1, n}\left(a, \prec^{*}\right)}=t^{p o s(b)+1} t^{\operatorname{comaj}_{n-k+1, n}\left(b, \prec^{*}\right)-1}=t^{p o s(b)} t^{\operatorname{comaj}_{n-k+1, n}\left(b, \prec^{*}\right)}
$$

as desired.

Thus our involution $J_{k}$ shows that

$$
\begin{aligned}
& \sum_{a=a_{1} \cdots a_{n} \in A_{ \pm}^{n}} t^{\text {pos(a)}(-1)^{n e g(a)}} z_{\left|a_{1}\right|} \cdots z_{\left|a_{n}\right|} q^{\operatorname{maj}_{1, n-k+1}\left(a, \prec^{*}\right)} t^{\operatorname{comaj}_{n-k+1, n}\left(a, \prec^{*}\right)} \\
= & \sum_{a=a_{1} \cdots a_{n} \in A_{ \pm}^{n}, J_{k}(a)=a} t^{p o s(a)}(-1)^{n e g(a)} z_{\left|a_{1}\right|} \cdots z_{\left|a_{n}\right|} q^{\operatorname{maj}_{1, n-k+1}\left(a, \prec^{*}\right)} t^{\operatorname{comaj}_{n-k+1, n}\left(a, \prec^{*}\right)} .
\end{aligned}
$$

But since the only words $a=a_{1} \cdots a_{n}$ such that $J_{k}(a)=a$ must have $\left|a_{n-k+1}\right| \cdots\left|a_{n}\right|$ be pairwise distinct, it follows that the largest possible type of a monomial $z_{\left|a_{1}\right|} \cdots z_{\left|a_{n}\right|}$ relative to the dominance order is $\left(1^{k-1}, n-k+1\right)$ since this is largest type of words with at least $k$ distinct letters. Thus (43) holds.

\section{Final Remarks}

\subsection{Haglund Statistics}

Let $\xi$ be a filling of the Ferrers diagram of a partition $\mu$ with the numbers $1, \ldots, n$. For any cell $u=(i, j) \in F_{\mu}$, let $\xi(u)$ be the entry in cell $u$. We say that $u=(i, j) \in F_{\mu}$ is a descent of $\xi$, written $u \in \operatorname{Des}(\xi)$, if $i>1$ and $\xi((i, j)) \geq \xi((i-1, j))$. Then $\operatorname{maj}(\xi)=\sum_{u \in \operatorname{Des}(\xi)}(\operatorname{leg}(u)+1)$. Two cells $u, v \in F_{\mu}$ attack each other if either

(a) they are in the same row, i.e. $u=(i, j)$ and $v=(i, k)$, or 


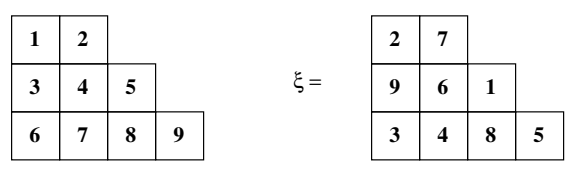

reading order

Figure 7: The reading order and a filling of $(4,3,2)$.

(b) they are in consecutive rows, with the cell in the upper row strictly to the right of the one in the lower row, i.e. $u=(i+1, k)$ and $v=(i, j)$ where $j<k$.

The reading order is the total ordering on the cells of $F_{\mu}$ given by reading the cells row by row from top to bottom, and left to right within each row. For example, the reading order of $(2,3,4)$ is depicted on the left in Figure 7 . An inversion of $\xi$ is a pair of entries $\xi(u)>\xi(v)$ where $u$ and $v$ attack each other and $u$ precedes $v$ in the reading order. We then define $\operatorname{Inv}(\xi)=\{\{u, v\}: \xi(u)>\xi(v)$ is an inversion $\}$ and $\operatorname{inv}(\xi)=|\operatorname{Inv}(\xi)|-\sum_{u \in \operatorname{Des}(\xi)} \operatorname{arm}(u)$.

For example, if $\xi$ is the filling of shape $(2,3,4)$ depicted in Figure 7 , then Des $(\xi)=$ $\{(2,1),(2,2),(3,2)\}$. There are four inversion pairs of type (a), namely $\{(2,1),(2,2)\}$, $\{(2,1),(2,3)\},\{(2,2),(2,3)\}$, and $\{(1,3),(1,4)\}$, and one inversion pair of type (b), namely $\{(2,2),(1,1)\}$. Then one can check that $|\operatorname{Inv}(\xi)|=5, \operatorname{maj}(\xi)=5$ and $\operatorname{inv}(\xi)=2$. Finally, we can identify $\xi$ with a permutation by reading the entries in the reading order. In the example of Figure $7, \xi=279613485$. Then we let $D(\xi)=\operatorname{Des}\left(\xi^{-1}\right)$. In our example, $\xi^{-1}=516794283$ so that $D(\xi)=\{1,5,6,8\}$.

Recently, Haglund, Haiman and Loehr [20, 21] proved Haglund's conjectured combinatorial interpretation [19] of $\tilde{H}_{\mu}(\bar{x} ; q, t)$ in terms of quasi-symmetric functions. That is, given a non-negative integer $n$ and a subset $D \subseteq\{1, \ldots, n-1\}$, Gessel's quasi-symmetric function of degree $n$ in variables $x_{1}, x_{2}, \ldots$ is defined by the formula

$$
Q_{n, D}(\bar{x}):=\sum_{\substack{a_{1} \leq a_{2} \leq \cdots \leq a_{n} \\ a_{i}=a_{i+1} \Rightarrow i \notin D}} x_{a_{1}} x_{a_{2}} \cdots x_{a_{n}} .
$$

Then Haglund, Haiman and Loehr [21] proved

$$
\tilde{H}_{\mu}(\bar{x} ; q, t)=\sum_{\xi: \mu \simeq\{1, \ldots, n\}} q^{i n v(\xi)} t^{\operatorname{maj}(\xi)} Q_{n, D(\xi)}(\bar{x}) .
$$

Here the sum runs over all fillings $\xi$ of the Ferrers diagram of $\mu$ with the numbers $1, \ldots, n$.

\subsection{Relations with the Combinatorial Interpretation of Macdon- ald Polynomials}

The Hilbert series of $\mathbf{H}_{\mu}$ is equal to the coefficient of $x_{1} x_{2} \cdots x_{n}$ in $\tilde{H}_{\mu}(\bar{x} ; q, t)$. Since the coefficient of $x_{1} x_{2} \cdots x_{n}$ in any quasi-symmetric function $Q_{n, D}(\bar{x})$ is 1 , it follows that the 
Hilbert series of $\mathbf{H}_{\mu}$ is given by

$$
\sum_{k, r} \operatorname{dim} \mathbf{H}_{\mu}^{(h, k)} q^{h} t^{k}=\left.\tilde{H}_{\mu}(\bar{x} ; q, t)\right|_{x_{1} x_{2} \cdots x_{n}}=\sum_{\xi: \mu \simeq\{1, \ldots, n\}} q^{i n v(\xi)} t^{\operatorname{maj}(\xi)},
$$

where the sum runs over all fillings $\xi$ of the Ferrers diagram of $\mu$ with the numbers $1, \ldots, n$. No known basis realizes this remarkable identity for general $\mathbf{H}_{\mu}$. The $k$-th Haglund basis described in Subsection 1.3.2 above provides such a basis when $\mu$ is of hook shape.

\section{References}

[1] R. M. Adin, F. Brenti and Y. Roichman, Descent representations and multivariate statistics, Trans. Amer. Math. Soc. 357 (2005), 3051-3082.

[2] R. M. Adin, A. Postnikov and Y. Roichman, Hecke algebra actions on polynomial rings, J. Algebra 233 (2000), 594-613.

[3] R. M. Adin, A. Postnikov and Y. Roichman, On characters of Weyl groups. Discrete Math. 226 (2001), 355-358.

[4] E. E. Allen, The descent monomials and a basis for the diagonally symmetric polynomials, J. Alg. Combin. 3 (1994), 5-16.

[5] E. E. Allen, Bitableaux bases for the diagonally invariant polynomial quotient rings, Adv. Math. 130 (1997), 242-260.

[6] J.-C. Aval, Monomial bases related to the $n$ ! conjecture, Discrete Mathematics 224, (2000), 15-35.

[7] H. Barcelo, Young straightening in a quotient $S_{n}$-module, J. Alg. Combin. 2 (1993), $5-23$.

[8] A. Berele and A. Regev, Hook Young diagrams and their applications to combinatorics and to representations of Lie superalgebras, Adv. in Math. 64 (1987), 118-175.

[9] A. Berele and J. B. Remmel, Hook flag characters and their combinatorics, J. Pure Appl. Alg. 35 (1985), 225-245.

[10] N. Bergeron, F. Bergeron, A. M. Garsia, M. Haiman, and G. Tesler, Lattice diagram polynomials and extended Pieri rules, Adv. Math. 142 (1999), 244-334.

[11] A. M. Garsia and M. Haiman, A graded repesentation model for the Macdonald polynomials, Proc. Nat. Acad. Sci. 90 (1993), 3607-3610.

[12] A. M. Garsia and M. Haiman, Factorizations of Pieri rules for Macdonald polynomials, Discrete Math. 139 (1995), 219-256.

[13] A. M. Garsia and M. Haiman, Some natural bigraded $S_{n}$-modules, Electronic Journal of Combinatorics 3 (1996), R24.

[14] A. M. Garsia and M. Haiman, Orbit Harmonics and Graded Representations, Research Monograph to appear as part of the collection published by the Lab. de. Comb. et Informatique Mathématique, edited by S. Brlek, U. du Québec á Montréal. 
[15] A. M. Garsia and C. Procesi, On certain graded $S_{n}$-modules and the $q$-Kostka polynomials, Adv. Math. 94 (1992), 82-138.

[16] A. M. Garsia and J. Remmel, Shuffles of permutations and the Kronecker product, Graphs and Combinatorics 1 (1985), 217-263.

[17] A. M. Garsia and D. Stanton, Group actions of Stanley-Reisner rings and invariants of permutation groups, Adv. Math. 51 (1984), 107-201.

[18] I. M. Gessel, Multipartite P-partitions and inner products of Schur functions, Contemp. Math. 34 (1984), 289-302.

[19] J. Haglund, A Combinatorial Model for the Macdonald polynomials, Proc. Nat. Acad. Sci. 101 (2004), 16127-16131.

[20] J. Haglund, M. Haiman, and N. Loehr, Combinatorial theory of Macdonald polynomials I: Proof of Haglund's formula, Proc. Nat. Acad. Sci. 102 (2005), 2690-2696.

[21] J. Haglund, M. Haiman, and N. Loehr, A Combinatorial formula for the Macdonald polynomials, J. Amer. Math. Soc. 18 (2005), 735-761.

[22] M. Haiman, Macdonald polynomials and geometry, in: New Perspectives in algebraic geometry (Berkeley, CA, 1996-97), Math. Sci. Res. Inst. Publ., vol. 38, pp. 207-254, Cambridge Univ. Press, Cambridge (1999).

[23] M. Haiman, Hilbert schemes, polygraphs, and the Macdonald positivity conjecture, J. Amer. Math. Soc. 14 (2001), 941-1006.

[24] J. E. Humphreys, Reflection Groups and Coxeter Groups, Cambridge Studies in Advanced Mathematics 29, Cambridge Univ. Press, Cambridge, 1990.

[25] D. Kazhdan and G. Lusztig, Representations of Coxeter groups and Hecke algebras, Invent. Math. 53 (1979), 165-184.

[26] D. E. Knuth, Permutations, matrices and generalized Young tableaux, Pacific J. Math. 34 (1971), 709-727.

[27] I. G. Macdonald, A new class of symmetric functions, Publ. I.R.M.A. Strasbourg, Actes $20^{e}$ Séminaire Lotharingien (1988), 131-171.

[28] I. G. Macdonald, Symmetric Functions and Hall Polynomials, $2^{\text {nd }}$ ed., Oxford Univ. Press (1995).

[29] A. Regev and T. Seeman, Shuffle invariance of the super-RSK algorithm, Adv. Appl. Math. 28 (2002), 59-81.

[30] J. B. Remmel, The combinatorics of $(k, l)$-hook Schur functions, Contemp. Math. $A M S$, vol. 34 (Comb. and Alg.) (1984), pp. 253-287.

[31] J. B. Remmel, A bijective proof of a factorization theorem for $(k, l)$-hook Schur functions, Lin. and Multilin. Alg. 28 (1990), 253-287.

[32] G. de B. Robinson, On the representations of $S_{n}$, Amer. J. Math. 60 (1938), 745-760.

[33] C. E. Schensted, Longest increasing and decreasing subsequences, Canad. J. Math. 13 (1961), 179-191. 
[34] B. Sagan, The Symmetric Group: Representations, Combinatorial Algorithms, and Symmetric Functions, Wadsworth and Brooks/Cole, 1991.

[35] L. Solomon, The orders of the finite Chevalley groups, J. Algebra 3 (1966), 376-393.

[36] R. P. Stanley, Invariants of finite groups and their applications to combinatorics, Bull. Amer. Math. Soc. (new series) 1 (1979), 475-511.

[37] R.P. Stanley, Some aspects of group acting on finite posets, J. Combin. Theory Ser. A 32 (1982), 132-161.

[38] J. R. Stembridge, Some particular entries of the two-pararemeter Kostka matrix, Proc. Amer. Math. Soc. 121 (1994), 469-490.

[39] R. Steinberg, On a theorem of Pittie, Topology 14 (1975), 173-177. 